\title{
Single-cell Chromatin Accessibility and Lipid Profiling Reveals a Metabolic Shift in Adipocytes Induced by Bariatric Surgery
}

\author{
Blaine Harlan ${ }^{1}$ \\ Hui Gyu Park² \\ Roman Spektor ${ }^{1,6}$ \\ Bethany Cummings 3,4 \\ J. Thomas Brenna ${ }^{2,5}$ \\ Paul D. Soloway ${ }^{1,4,5}$
}

${ }^{1}$ Field of Genetics, Genomics, and Development, Department of Molecular Biology and Genetics, Cornell University, Ithaca, New York 14853, USA.

${ }^{2}$ Dell Pediatric Research Institute, Dept of Pediatrics, University of Texas at Austin, 1600 Barbara Jordan Blvd, Austin, Tx 78701

${ }^{3}$ Department of Surgery, School of Medicine, University of California, Davis, Sacramento, CA ${ }^{4}$ Department of Biomedical Sciences, College of Veterinary Medicine, Cornell University, Ithaca, New York 14853, USA.

${ }^{5}$ Division of Nutritional Sciences, College of Agriculture and Life Sciences, Cornell University, Ithaca, New York 14853, USA.

${ }^{6}$ Present address, CRISPR Therapeutics 


\begin{abstract}
Obesity promotes type 2 diabetes and cardiometabolic pathologies. Vertical sleeve gastrectomy (VSG) is used to treat obesity resulting in long-term weight loss and health improvements that precede weight loss; however, the mechanisms underlying the immediate benefits remain incompletely understood. Because adipose plays a crucial role in energy homeostasis and utilization, we hypothesized that VSG exerts its influences, in part, by modulating adipose functional states. We applied single-cell ATAC sequencing and lipid profiling to inguinal and epididymal adipose depots from mice that received sham surgery or VSG. We observed depotspecific cellular composition and chromatin accessibility patterns that were altered by VSG. Specifically, accessibility at Scd1, a fatty acid desaturase, was substantially reduced after VSG in mature adipocytes of inguinal but not epididymal depots. This was accompanied by reduced accumulation of SCD1-produced unsaturated fatty acids. Given these findings and reports that reductions in Scd1 attenuate obesity and insulin resistance and that unsaturated fatty acids stimulate glucose uptake, storage, and oxidation, our results suggest VSG exerts its beneficial effects through modifications of fatty acid profiles mediated by Scd1.
\end{abstract}




\section{Introduction}

In obesity, the positive energy balance can lead to insulin resistance, chronic inflammation, cardiovascular disease, and dyslipidemia. Currently, vertical sleeve gastrectomy (VSG) is the most common bariatric surgical treatment for obesity in humans, and many effects seen in humans have been demonstrated in mouse models. VSG leads to rapid metabolic changes that precede weight loss; notably, $80 \%$ of patients with type 2 diabetes experience complete remission after bariatric surgery, often within days (Buchwald et al., 2004; Pories et al., 1995). Bariatric surgery remains the most effective treatment for long-term remission of type 2 diabetes (Zhang et al., 2014). The mechanisms underlying weight loss-independent improvements are incompletely characterized. One may involve liver bile acids, which regulate glucose homeostasis through the bile acid receptor TGR5. Signaling through this pathway increases after VSG in mice (McGavigan, Garibay, et al., 2017). Additionally, levels of GLP-1, a hormone produced by the gut, are increased after VSG, and its signaling through GLP-1R in pancreatic $\beta$ cells is important for the glucoregulatory improvements of VSG (Garibay et al., 2018). There is extensive crosstalk between the liver and adipose tissue, which affects the metabolism of glucose and lipids. However, the effects of VSG on adipose depots specifically have not been thoroughly characterized.

Adipose depots are found throughout the body and play different roles in metabolism. In humans, white adipose depots classified based on location include visceral adipose tissue (VAT) or subcutaneous adipose tissue (SAT). Excess VAT has been associated with many health risks, while excess SAT lacks these deleterious associations.

Bariatric surgery has been reported to impart several changes to adipose tissue. There is a loss of fat mass, first in SAT and later in VAT, indicating the depots respond differentially to the intervention (Galanakis et al., 2015; Toro-Ramos et al., 2015; Yoon et al., 2007). After VSG, levels of adiponectin rise; its elevated autocrine and endocrine activities can respectively increase fatty acid oxidation in adipocytes and inhibit liver glucose production (Sowers, 2008). Reduced fat mass after VSG normalizes leptin levels, which can lead to reduced food intake. Metabolic shifts in SAT and VAT adipose tissue have been reported after VSG (Harris et al., 2020; Jahansouz et al., 2018). One study found increased lipolysis, lipid oxidation, and thermogenic gene expression in SAT from human patients. Some of these metabolic changes are consistent with stimulation of adipose beiging, in which white adipocytes become more similar to brown adipocytes leading to increased lipolysis, $\beta$-oxidation, and thermogenesis. Beige adipose thermogenesis, the production of heat by cellular metabolism, can increase basal energy expenditures leading to a negative energy balance, weight loss, and restoration of a healthy metabolism. While VSG alters lipid usage, the expression of UCP1, a critical factor involved in thermogenesis, appears to remain unchanged (Harris et al., 2020; Jahansouz et al., 2018). However, UCP1-independent thermogenesis pathways have been described, leaving open the possibility that beiging occurs within white adipose tissue after VSG (Ikeda et al., 2017; Ukropec et al., 2006). Complicating these analyses are regional differences in the beiging process within the same adipose depot and between depots (Chi et al., 2018). Overall, the mechanisms by which VSG influences adipose functions are incompletely characterized.

Because the increased energy expenditure induced by VSG may occur through non-shivering thermogenesis, we hypothesized that VSG induces changes in lipid metabolism in adipose and imparts other cell state changes in adipose tissues, particularly in mature adipocytes. Given the functional differences among adipose depots and the cell heterogeneity within depots, an 
understanding of the effects of VSG on adipose function requires high-resolution analyses at the cellular level.

Tissue analyses at the single-cell level can identify cell types and report changes in cellular composition and chromatin regulation resulting from VSG interventions. Most single-cell sequencing technologies rely on microfluidic devices, which are unsuitable for assaying large lipid-filled mature adipocytes, given their buoyancy in aqueous media. Chromatin accessibility in isolated nuclei can reveal, in an unbiased way, the range of cell types present in adipose depots, their abundances, and changes in their regulatory sites that arise in response to VSG. We applied this analysis to inform the mechanisms by which VSG modifies adipose and restores healthy energy homeostasis.

Our study investigated the chromatin accessibility changes in single cells of inguinal (subcutaneous) and epididymal (visceral) adipose depots after VSG or sham surgery. We identified distinct cellular compositions and gene accessibilities between these depots.

Additionally, we characterized three subtypes of mature adipocytes in both depots and identified differences in abundance and chromatin accessibility that varied with depot and surgical treatment. Altered chromatin states were observed in inguinal VSG-responsive adipocytes at genes regulating lipid metabolism. These included genes essential to $\beta$-oxidation of lipids and fatty acid saturation state. These chromatin changes were also accompanied by corresponding changes in unsaturated lipid biogenesis and a shift of white adipocytes to a thermogenic beigelike state. Previous studies have shown that these changes are protective against obesity and improve glucose metabolism, leading to the conclusion that VSG exerts its beneficial effects, in part, through modifying the metabolism of unsaturated fatty acids and increased energy expenditure through thermogenesis in a subset of adipocytes in the inguinal depot. 


\section{Results}

\section{snATAC libraries from inguinal and epididymal adipose tissue}

To characterize the effects of VSG on adipose tissues that are independent of body mass, we used tissues from mice fed a high-fat diet for eight weeks before being subjected to VSG. As controls, we used weight-matched sham-operated animals (fig 1a). The metabolic phenotyping for these mice has been previously reported in (McGavigan, Henseler, et al., 2017). The white adipose depots we used for chromatin and lipid profiling studies were inguinal (subcutaneous) and epididymal (visceral), collected from three animals each.

Prior single-cell sequencing studies using white adipose tissue focused on the stromal vascular fraction (SVF), which does not contain mature adipocytes (Vijay et al., 2020). Although the buoyancy created by lipids in mature adipocytes interferes with the reliable formation of aqueous droplets used in many single-cell methods, the combinatorial indexing approach using isolated nuclei in single nuclei ATAC-seq (snATAC-seq) allowed us to study the chromatin accessibility changes in adipose tissue from mice that underwent VSG. After sequencing the snATAC libraries, removing doublets, and applying quality control cutoffs, we acquired chromatin profiles from 28,372 cells, with a minimum of 5,087 cells per group in inguinal (INGSHAM, ING-VSG) and epididymal (EPI-SHAM, EPI-VSG) samples (fig 1b,1c, and S1). An advantage of ATAC-seq is the ability to examine chromatin accessibility at distal intergenic regions in addition to genes, which can identify potential regulatory elements that control cell properties and responses. We called peaks on aggregated data from each treatment group. About $25 \%$ of the peaks fell in promoter regions and $25 \%$ in distal regions (figure $1 \mathrm{~d}$ ). 


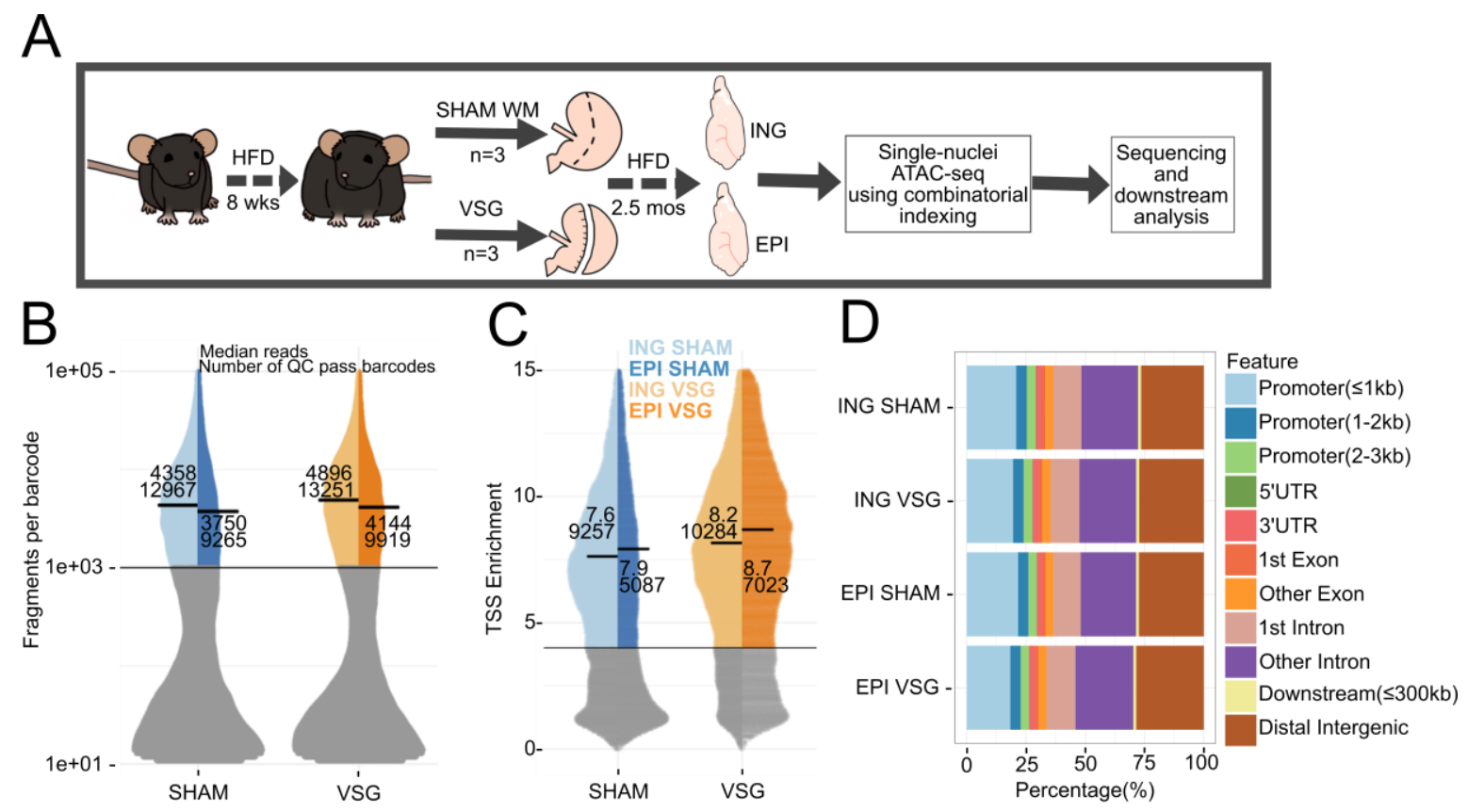

Figure 1. Experimental design and quality control metrics for single nuclei ATACseq.

(A) Experimental design showing details of treatments and adipose tissues used for snATAC. Sham mice were pair-fed to be weight matched (WM) to VSG animals.

(B) Fragment count distributions per barcode for each cell from SHAM- or VSG-derived depots. Cells with 1,000 or more fragments were kept for further analysis. The median fragment count for each group (top number) is indicated with a thick bar; lower number identifies the number of bar codes or cells passing that cutoff.

(C) TSS enrichment score analysis of cells with 1,000 or more fragments per cell. Cells with a score of 4 or higher were kept for further analysis. The median score for each group (top number) is indicated with a thick bar; lower number identifies the number of bar codes or cells passing that cutoff.

(D) Feature distribution of peaks called on aggregated data for each sample type.

\section{Clustering and cell type identification of cells within adipose depots reveals distinct adipocyte subtypes}

We first identified cell types by unsupervised clustering of the snATAC data after dimensional reduction. There were eleven clusters of cells in the combined ING and EPI dataset, representing 28,372 cells (fig 2a). To classify cell types within these clusters, we used gene ontology (GO) enrichment for the top 200 differentially accessible genes from each cluster to provide insight into biological processes (fig $2 b$ and fig S2c). We then confirmed the cell type assignment using known marker genes (fig 2c, fig 2d). Four clusters (AD1-4) collectively represented mature adipocytes, the most abundant cell type. Their top differentially accessible 
genes included canonical adipocyte markers such as Pparg and Cebpa and genes in GO enrichment categories that included fatty acid metabolic process.

The second largest cluster (APC) had GO enrichment categories associated with morphogenesis and WNT signaling. This cluster represented adipocyte progenitor cells (APCs) based on accessibility at known APC markers Fbn1, Timp2, Mfap5, Anxa3 (Cho et al., 2019). The next cluster (End), identified as endothelial cells, had signatures of angiogenesis and tight junction enrichments and known marker genes Cldn5, S1pr1, Pecam1, Ptprb, Kdr (Schupp et al., 2020). Two clusters of immune cells (Mac, Tcell) had distinct gene accessibility. In the first immune cluster, we found enrichment of the GO categories T-cell activation and protein localization to the endosome; endosomes are essential in regulating antigen receptor signaling (Gleeson, 2014). Among the top differentially accessible T-cell markers were Cd3d, Ikzf3, Sla, Itk, Cd8a. The second immune cluster had an enrichment for the macrophage migration GO term and higher accessibility in Mgl2, Csf1r, Ccl6, C1qc, Irf5.

One unidentified cluster (Unk) did not result in any significant GO enrichments and few differentially accessible genes. This cluster also had a lower mean TSS enrichment score than other clusters, suggesting this cluster represented lower quality nuclei with high background reads. Therefore, it was excluded from further analysis. Finally, we identified two clusters (P.Epi and B.Epi) as basal epididymal cells and principal epididymal cells based on marker genes (fig S2b) (Rinaldi et al., 2020). Because the epididymis is located close to the EPI depot, epididymal cells included in the EPI adipose are likely a dissection artifact, and they were not included in the analysis. While innervation in adipose tissue is vital in regulating adipose metabolism, cell body projections of neurons do not contain nuclei, and they were not present in the dataset. Overall, we found mature adipocytes, APCs, endothelial cells, T-cells, and macrophages. Since most of the cells within the dataset were mature adipocytes, this allowed us to examine regulatory changes at a single-cell level, which was not previously possible.

Among the four clusters of adipocytes, three (AD1, AD2, and AD4) had the properties of mature adipocytes, including high accessibility in canonical adipocyte markers Pparg and Cebpa. The remaining subcluster, AD3, had lower accessibility in Pparg than AD1,2, and 4, as well as accessibility in genes associated with non-adipocytes. Though AD3 shares some properties with the other three adipocytes, it was sufficiently distinct that we chose to exclude it from the analyses focused on mature adipocytes. AD3 is unlikely to be doublet cells as we previously removed likely doublets, and the abundance of likely doublets in these samples was what we expected from earlier analyses that directly measured doublet rates (data not shown). AD3 may be an intermediate cell type between APCs and mature adipocytes. Because that cluster displayed no abundance or accessibility differences between depots and treatment groups, we focused our attention on AD1,2,4 in our analyses of mature adipocytes.

To understand the molecular basis of adipocyte heterogeneity in the inguinal depot, we compared the accessibility of genes in AD1,2,4 (fig 2e). These clusters exhibited chromatin accessibility at marker genes for white adipose tissue, including Npr3, Adipoq, Lep, Fabp4, Nnat, Adcy5, Psat1, with the highest accessibility in AD2 and AD4. We also found that the beige-associated transcriptional regulators Prdm16 and Ebf2 were more accessible in AD1 (fig 2e, S4a). Notably, Prdm16 is a crucial transcriptional coregulator of the beiging and the thermogenic program (Cohen et al., 2014; Seale et al., 2011). Furthermore, Ebf2 transcriptionally regulates brown and beige adipocyte-specific genes by recruiting chromatin remodeling complexes (T. Liu et al., 2020; Rajakumari et al., 2013; Stine et al., 2016; Wang et 
al., 2014). Accessibility at Cox8b, a regulator of mitochondrial $\beta$-oxidation, was also increased in the AD1 cluster. These data suggest that among mature adipocytes, AD1 is competent to participate in thermogenesis, as is the case for beige adipocytes. While AD1 exhibits accessibility at both white and beige marker genes, most studies show beiging leads to a loss of the white adipocyte gene expression program rather than the acquisition of a beige program. Our findings might be explained by studies showing inguinal adipocytes can interconvert between white and beige functional states depending on environmental stimuli (Rosenwald et al., 2013). The maintenance of open chromatin within white marker genes in AD1 suggests that while these cells may be competent to participate in thermogenesis, they may also be poised to return to a white state in response to changing physiologic signals. Accessibility at bile acid receptor genes Gpbar1 (TGR5) and Nr1i3 (CAR) was not significantly different among any subclusters or treatment groups. Although TGR5 signaling is increased after VSG, this is due to increases in the bile acid ligands, which regulate glucose homeostasis through TGR5 (McGavigan, Garibay, et al., 2017). However, beige-like AD1 cells have increased accessibility of the TGR5 target genes (Prdm16, Cidea, and Ppargc1a) (fig 2e) (Velazquez-Villegas et al., 2018). 
bioRxiv preprint doi: https://doi.org/10.1101/2021.06.22.449469; this version posted June 22, 2021. The copyright holder for this preprint (which was not certified by peer review) is the author/funder, who has granted bioRxiv a license to display the preprint in perpetuity. It is made available under aCC-BY-NC-ND 4.0 International license.

A
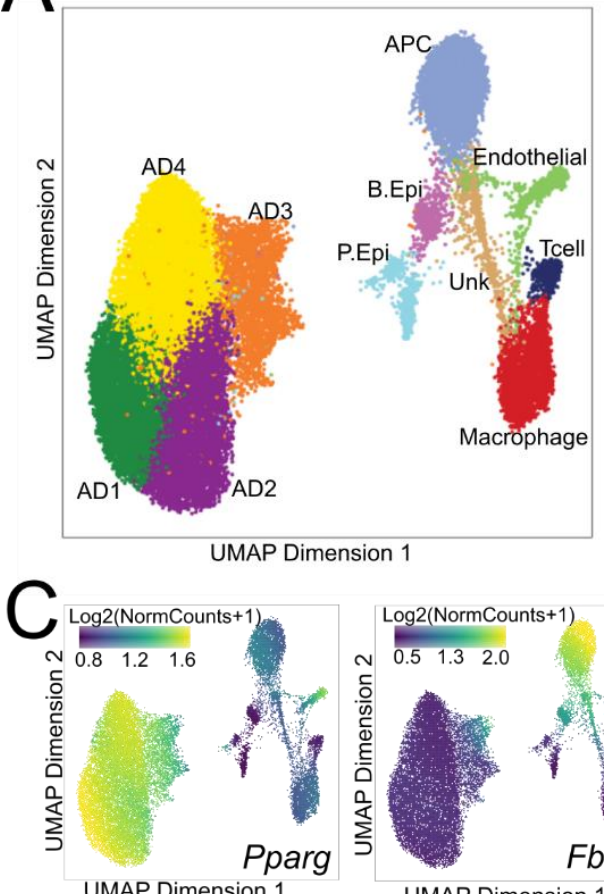

UMAP Dimension 1

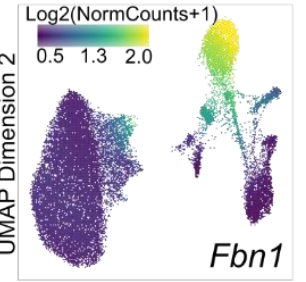

UMAP Dimension 1
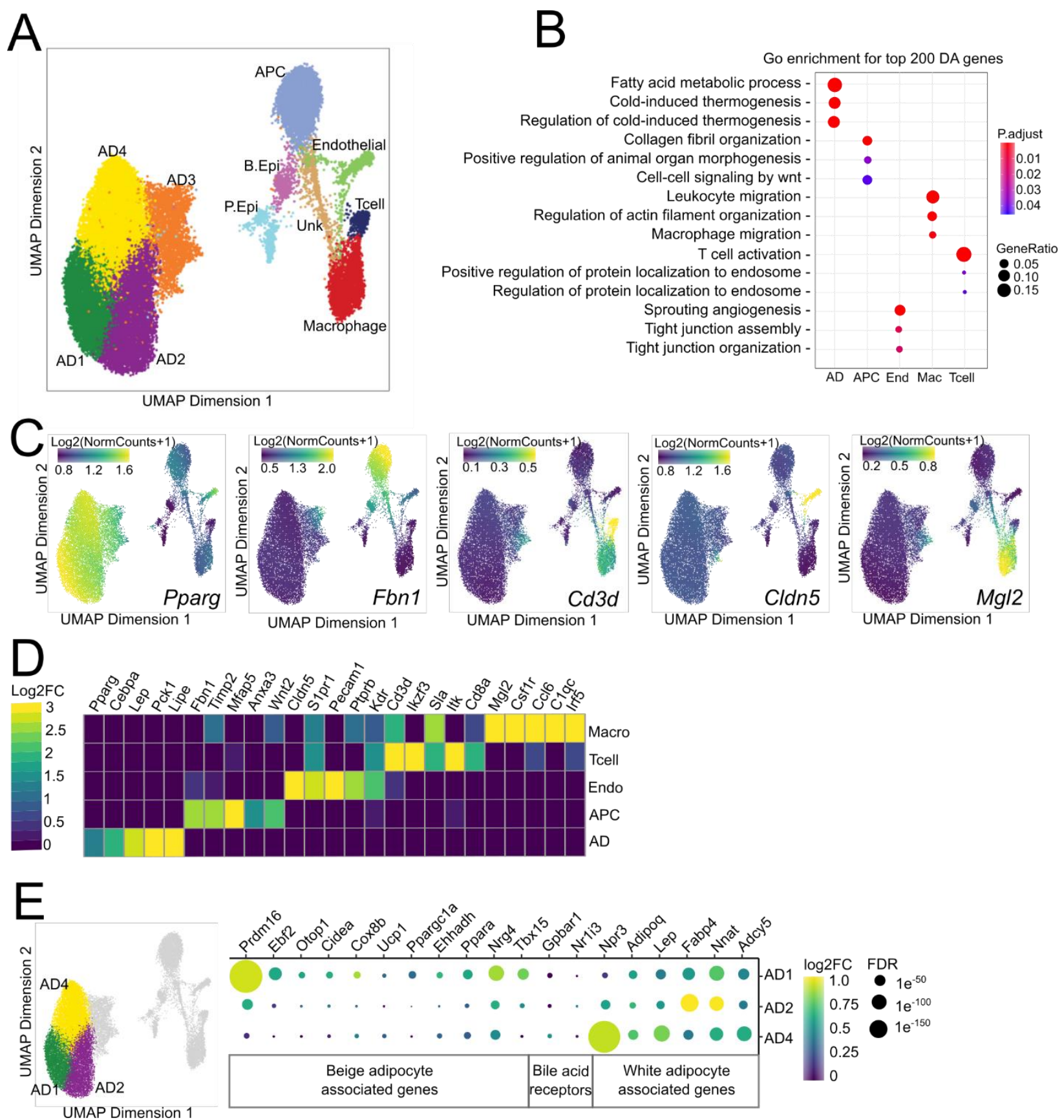
Figure 2. Clustering and cell type identification of cells in epididymal and inguinal adipose depots.

(A) UMAP plot of 28,372 cells passing quality control from inguinal and epididymal adipose tissues of VSG and SHAM mice. Colors represent 11 different clusters determined by unbiased clustering. APC, adipocyte progenitor cells; AD1, adipocyte subcluster 1; AD2, adipocyte subcluster 2; AD3, adipocyte subcluster 3; AD4, adipocyte subcluster 4; Mac, macrophage; Endo, Endothelial; Unk, unknown; B. Epi, basal epididymal epithelial; P. Epi, principal epididymal epithelial.

(B) Gene ontology enrichment for biological processes using the top 200 differentially accessible genes for each major cell type.

(C) Imputed marker gene accessibility using MAGIC overlaid on the UMAP plot.

(D) Validation of cell type annotation showing log2-fold change of selected cell type marker genes. FDR<0.01.

(E) Chromatin accessibility of beige (AD1) and white adipocyte (AD2, AD4) associated genes in the three mature adipocyte subclusters.

\section{Chromatin accessibility of developmental genes and cell type composition are adipose depot-specific}

Having identified the mature adipocytes in our data, we determined how they differ between the ING and EPI depots. Indeed, previous studies have identified significant functional heterogeneity between white adipose depots due to their divergent developmental origins (Kwok et al., 2016). We compared cluster abundances and accessibility patterns found in cells from SHAM animals to characterize the cellular and molecular differences between the depots. Comparison of the relative abundances of mature adipocytes within each cluster revealed AD1 to be 6.5-fold more abundant in ING relative to EPI, and AD4 2.3-fold more abundant in EPI relative to ING (fig 3a). The lack of beige-like AD1 in EPI is consistent with the reports that visceral fat depots are resistant to beiging (Wu et al., 2012). Because there were few AD1 cells in EPI, we restricted our comparisons of mature adipocytes between ING and EPI to AD2 and AD4. Many of the differentially accessible genes in AD4 between the two depots are associated with adipocyte differentiation, such as Zfhx4, Hoxa9, Hoxc10, Tbx15, and Mir196a/b (fig 3b). Hoxc10, which was more accessible in ING, has been shown to repress beiging in subcutaneous adipose tissue but is not expressed in brown or visceral adipose tissue ( $\mathrm{Ng}$ et al., 2017). Conversely, Mir196a, which was also more accessible in ING, was shown to increase expression of beige-associated genes through suppression of Hoxc8, suggesting AD4 adipocytes within the ING depot may be poised to regulate beiging (Mori et al., 2012). Another gene, Angpt/4, which was more accessible in ING AD2, promotes angiogenesis and allows expansion of subcutaneous depots in response to a positive energy balance (Gealekman et al., 2011).

In addition to finding differences in mature adipocytes within the two depots, we found differences within their precursors - the APC cluster. APCs were 1.5-fold more abundant in the EPI than the ING depot (fig 3c). Furthermore, there was depot-specific accessibility at established regulators of adipocyte development in APCs (fig $3 \mathrm{~d}$ ). Wt1, a selective marker of visceral APCs, was more accessible in EPI APCs, whereas Tbx15 was more accessible in ING APCs (Chau et al., 2014; Sanchez-Gurmaches et al., 2015). Notably, Tbx15 is required for 
beige cell emergence in white adipose tissue and priming of the thermogenic state (Ejarque et al., 2019; Sun et al., 2019). Collectively, the differences in accessibility of genes in APCs related to adipocyte differentiation highlight distinct mechanisms for regulating adipocyte precursors in the two depots. These same mechanisms are likely responsible for the observed difference in adipocyte abundance between ING and EPI depots and the greater propensity of cells in the ING depot to undergo beiging in response to physiologic stimuli, including VSG.

In addition to genes regulating adipocyte cell fate, we found gene accessibility changes in APCs that regulate interactions with immune cells. The adipokine $\mathrm{Cxc} / 14$, which was more accessible in ING APCs, is involved in recruiting anti-inflammatory, alternatively-activated (M2)

macrophages (Cereijo et al., 2018). In contrast, pro-inflammatory Tcf21 was more accessible in EPI APCs. These findings demonstrate limited inflammatory signals in the ING depot relative to the EPI depot. The macrophage cluster was more abundant in EPI adipose tissue; however, no genes were differentially accessible. There were no differences in abundance or accessibility of genes in T-cells or endothelial cells between the depots. 

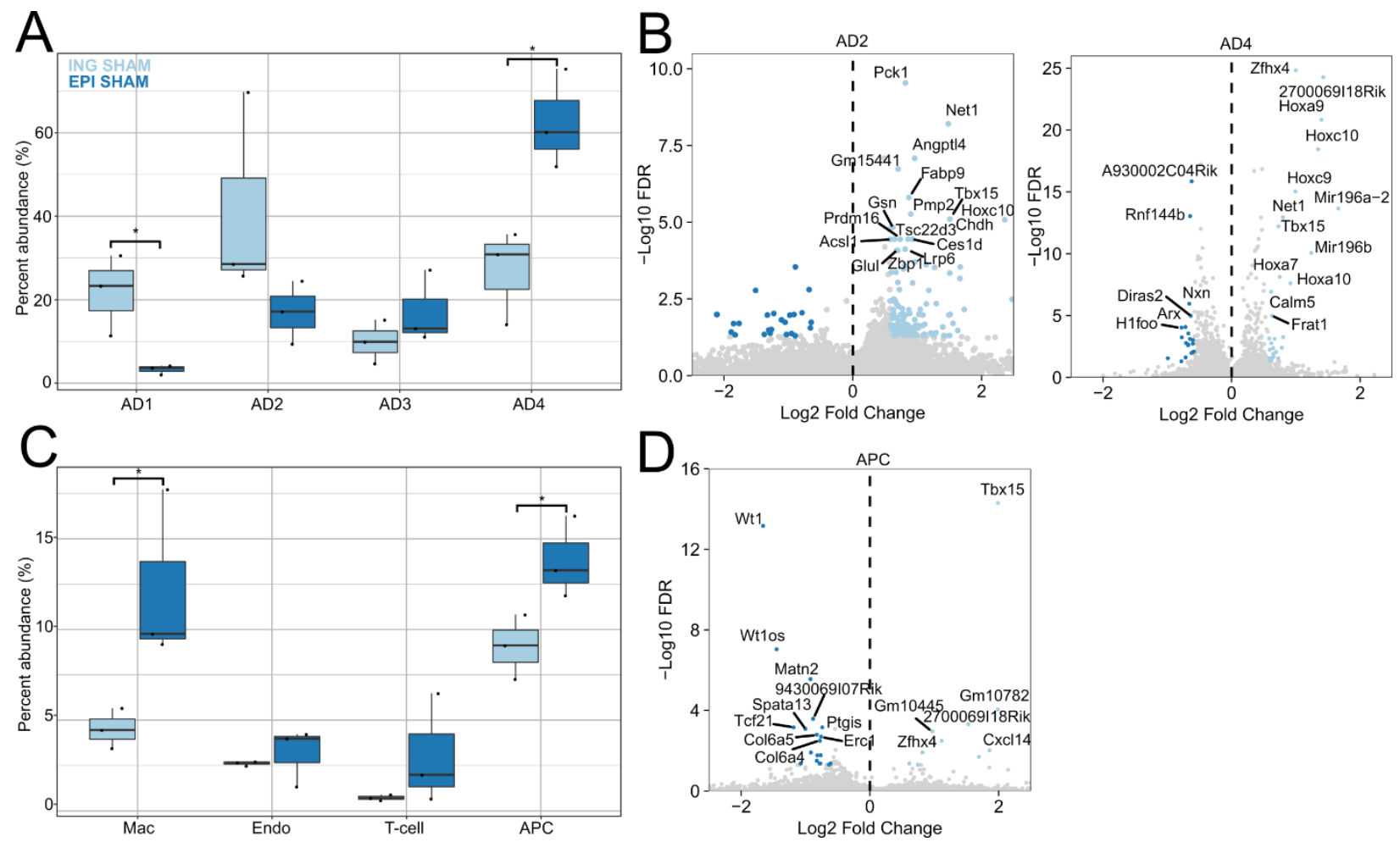

Figure 3. Adipose depot specific differences in cells from SHAM surgery mice

(A) Adipocyte abundances in ING (light blue) and EPI (dark blue) depots of SHAM animals. Dots represent each biological replicate. $(n=3)$

(B) Gene accessibility differences for AD2 (left) and AD4 (right) clusters comparing ING and EPI cells. Positive and negative log2-fold changes respectively indicate increased accessibility in ING and EPI depots.

(C) Abundances of endothelial, APC, T-cells and macrophages in ING (light blue) and EPI (dark blue) depots of SHAM animals $(n=3)$.

(D) Gene accessibility differences for APCs ING and EPI depots. Positive and negative log2-fold changes respectively indicate increased accessibility in ING and EPI depots.

${ }^{*} p<0.05$. Two-tailed students t-test was performed for $(A),(C)$.

\section{Changes in accessibility at transcription factor motifs identify regulatory changes in inguinal VSG adipocytes}

We next analyzed the effects of VSG on both cell abundances and transcription factor (TF) motif enrichments at sites of differential accessibility. The latter focus was motivated by the goal of understanding the regulatory mechanisms underlying VSG responses. Overall, the distribution of cell abundances in clusters did not change significantly between VSG and SHAM in either 
depot (fig S4b). While adipocyte progenitor cells and macrophages are known to change in response to obesity, we observed few cell number or accessibility changes between VSG and SHAM cells in ING and EPI adipose tissue for these cell types or T-cells. This may indicate that immune cells and APCs are not involved in physiologic changes associated with VSG. However, these cells represented fewer than $15 \%$ of the total cells analyzed from the four groups of mice, and accordingly, subtle but relevant changes may not be revealed in our data.

To identify potential gene regulatory changes that accompany VSG, we first characterized the transcription factor motifs enriched in adipocytes from the ING and EPI depots at regions of differentially accessible chromatin. For these analyses, we utilized the combined data from all classes of adipocytes, as limiting the analyses to individual subsets of adipocytes reduced our overall power. We first called peaks on the combined dataset, then determined differentially accessible peaks in VSG and SHAM-ING adipocytes. We found 13,449 peaks with increased accessibility and 4,943 with decreased accessibility in VSG mice (fig 4a). In peaks with increased accessibility, there were enrichments for STAT5A/B, NFIC/X, and STAT1,3,4 motifs (fig 4b and 4c). Importantly, adipocytes express STAT1,3,5A, 5B, and 6; therefore, the differential accessibility at motifs for those factors is likely to have functional consequences (Zhao \& Stephens, 2013). Notably, STAT5A directly binds to the promoter of Acox1, the first enzyme of the fatty acid $\beta$-oxidation pathway, which catalyzes the desaturation of acyl-CoAs to 2-trans-enoyl-CoAs (Coulter \& Stephens, 2006; W. Liu et al., 2020). This suggests that among the effects of VSG is elevated Stat5-dependent fatty acid $\beta$-oxidation. In peaks with decreased accessibility in adipocytes from VSG mice, there was an enrichment of motifs for the progesterone receptor (PGR) and the glucocorticoid receptor (NR1C3) (fig 4b). Consistent with this, in VSG mice, we found reduced accessibility within genes known to be regulated by glucocorticoids such as Serpine1, Fkbp5, Scd1 (fig 5a). Because glucocorticoids are associated with stress responses and promote obesity and insulin resistance, this further suggests that additional effects of VSG include reduction in glucocorticoid-mediated stress responses and altered regulation of glucocorticoid target genes that influence metabolism.

Transcription factor motifs enriched at differentially accessible peaks indicate their potential for regulating nearby target genes. To determine which genes could be regulated by the top enriched motifs, we located genes within $100 \mathrm{~kb}$ of differentially accessible peaks containing STAT5A, NFIC, and NR1C3 motifs and then identified the biological processes they control as indicated by GO enrichment (fig 4d). Surprisingly, each TF motif resulted in different GO enrichment categories for VSG and SHAM, indicating that coregulatory factors may modulate transcription factor binding under the physiological conditions affected by VSG, and that the coregulators may shift the effects of the TFs. In adipocytes from mice subjected to VSG, GO enrichments at putative target genes near sites of increased STAT5A, NFIC, and NR1C3 motif accessibility were associated with adaptive thermogenesis, cold-induced thermogenesis, and fatty acid oxidation. Notably, we observed several peaks with STAT5A motifs near Elovl5 (fig S3b), a fatty acid elongase. In contrast, genes near peaks with significantly decreased accessibility were associated with inflammation (inflammatory response, interleukin-1 mediated signaling pathway, stress-activated MAPK cascade) GO enrichment categories. The genes associated with STAT5A SHAM peaks did not produce significant GO enrichments. These findings are consistent with the facts that improvements in metabolism and reductions in inflammation are associated with VSG, and they strongly implicate the functional importance of these specific TFs in adipose homeostasis impacted by VSG. 
TF motif enrichment identifies potential TF binding regions, not all of which are bound.

Therefore, we sought to validate whether regions with NR1C3 motifs were indeed bound by the glucocorticoid receptor (GR) in adipocytes and if there were differences between the treatment groups. Yu et al. identified GR binding regions in 3T3-L1 differentiated adipocytes using ChIPseq (Yu et al., 2010). They found that many of the GR binding sites were near genes involved in triglyceride homeostasis. Several of these were differentially accessible in our dataset, including Scd1 (fig 5a). To determine whether the motifs we found at open chromatin in adipose overlap with known GR binding regions in 3T3-L1 adipocytes, we first identified the subset of the top three thousand peaks in ING adipose that were both differentially accessible in VSG vs. SHAM mice and that had NR1C3 motifs, and then we intersected them with the GR ChIP-seq data from 3T3-L1 adipocytes. We found that 5.8\% of peaks with NR1C3 motif enrichment in SHAM adipose overlapped with GR binding regions in 3T3-L1 adipocytes, whereas only $0.8 \%$ of the VSG peaks overlapped (fig 4e). This difference in overlap suggests that accessible NR1C3 motifs in SHAM adipose are likely to include more functional GR binding sites than VSG adipose. Additionally, this difference may in fact understate the importance of GR binding sites in SHAM adipose, as the top peaks in SHAM adipose had more NR3C1 motifs $(4.5 \% \geq 2$ motifs) than did VSG adipose ( $1 \% \geq 2$ motifs). While in vitro cultured 3T3-L1 adipocytes do not replicate cell states found in adipose tissue, these findings are consistent with our interpretation that decreased gene binding and activation by GR is associated with adipocyte responses to VSG in vivo, linking this change in GR regulation with the beneficial effects of VSG. 

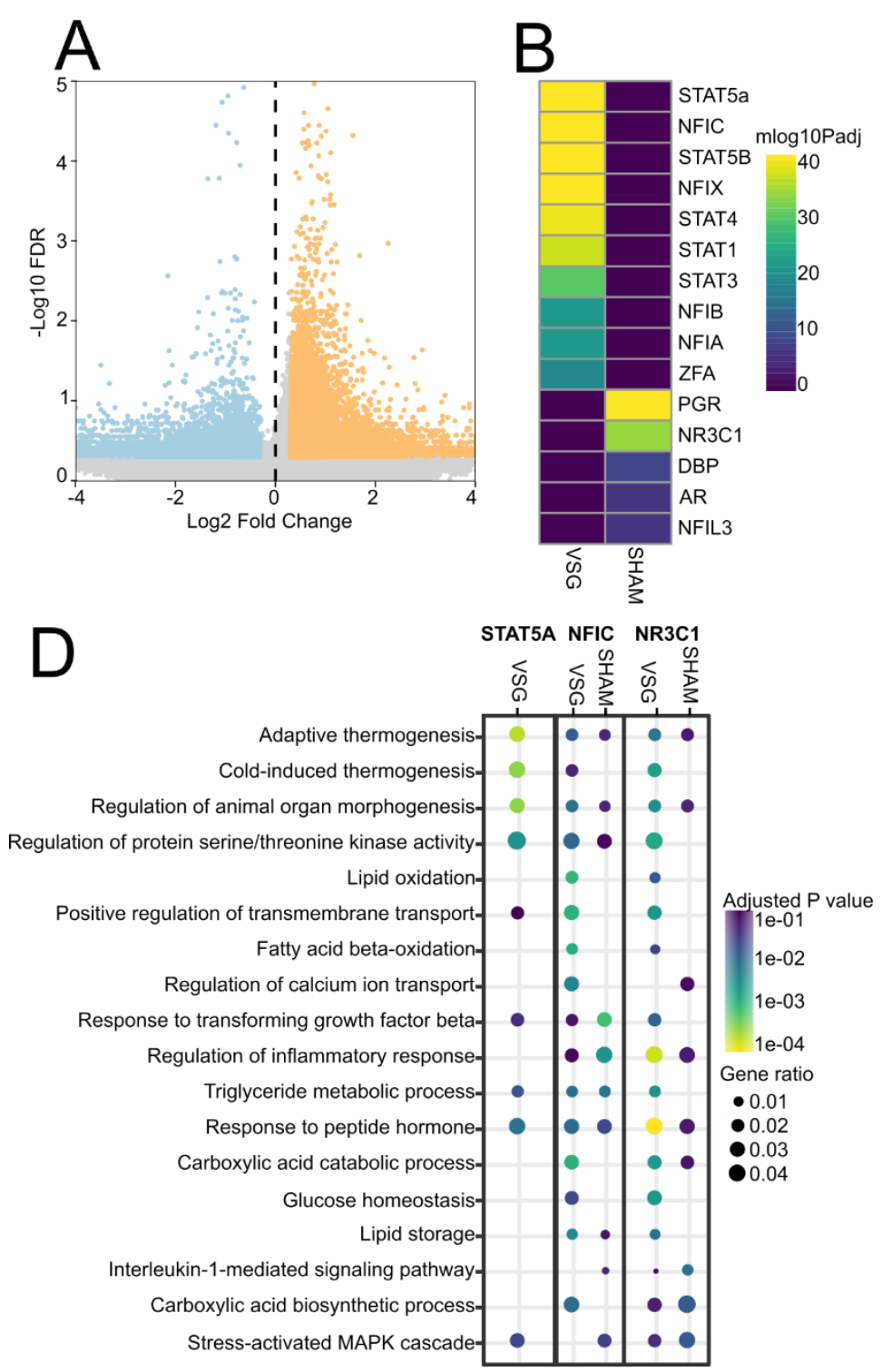
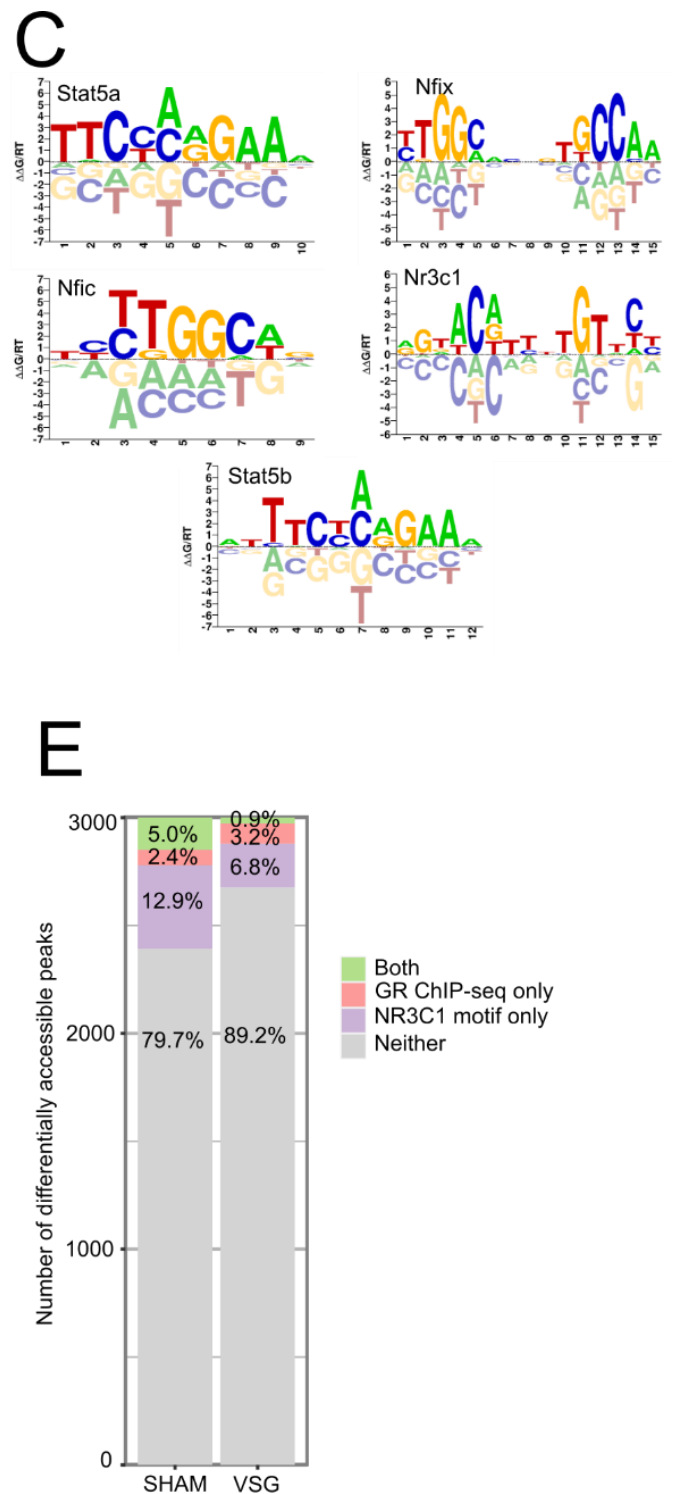

Figure 4. Differential accessibility of peaks with transcription factor motifs in response to VSG

(A) Volcano plot of differentially accessible peaks comparing VSG to SHAM ING adipocytes. The 13,449 peaks with positive fold change indicate increased accessibility in VSG; the 4,943 peaks with negative fold change indicate decreased accessibility in VSG. FDR $<0.5$ and absolute Log2Fold Change> 0.3.

(B) Transcription factor motif enrichment in differentially accessible peaks using the CISBP motif database. VSG (peaks with positive fold change) and SHAM (peaks with negative fold change).

(C) Motif sequence logos for the top enriched motifs.

(D) Gene ontology enrichment for biological process using the nearest genes $(<100 \mathrm{~kb})$ to peaks with top motifs. Adjusted P.value $<0.1$ for GO enrichments.

(E) Overlap of the top 3,000 differentially accessible peaks for VSG and SHAM ING adipocytes containing NR3C1 motifs, and glucocorticoid receptor ChIP-seq binding regions reported in Yu et al., 2010. 


\section{VSG induces gene accessibility changes in ING mature adipocytes regulating thermogenesis, insulin sensitivity, and lipid metabolism}

Many of the transcription factor motifs that were differentially accessible at intergenic sites of mature adipocytes from VSG mice, including those associated with GR binding, were found near genes essential to adipocyte metabolism. Therefore, we extended our analyses to consider additional functions of the genes nearest the differentially accessible peaks and to consider intragenic differential chromatin accessibility in adipocytes. Among the most differentially accessible genes in VSG vs. SHAM adipocytes were Adipor2, Pik3r1, Adrb3, Adcy5, which were significantly more accessible in VSG adipocytes, and Fkbp5, Serpine1, Scd1, Lpin1, which were significantly more accessible in SHAM adipocytes (fig 5a). Each of these genes has been implicated in the metabolic regulation of adipose. Their differential accessibility identifies pathways such as fatty acid and lipid metabolism that are influenced by VSG and likely mediate its effects (fig 5b).

After bariatric surgery, the well-studied adipokine, adiponectin, is increased in the plasma, and its receptor, Adipor2, is also upregulated in SAT (Savu et al., 2009). Our finding that VSG increases Adipor2 accessibility is consistent with these results and supports an autocrine role of adiponectin in improved adipose insulin sensitivity (Cummings et al., 2012; Kelly et al., 2016; Savu et al., 2009). Pik3r1, encoding a regulatory subunit of PI3K, is required for insulin signaling transduction, and accordingly, important for insulin sensitivity. Increased accessibility in these two insulin-sensitizing genes suggests a role for the ING depot in the improved insulin responsiveness after VSG. The sympathetic nervous system activates the $\beta-3$ adrenergic receptor, ADRB3, promoting beiging within white adipose tissue. ADRB3, but not ADRB1, stimulates a shift of mature white adipocytes into a beige state instead of de novo adipogenesis of beige adipocytes (Jiang et al., 2017). The increased accessibility at Adrb3 in adipocytes from VSG mice implicates its role, and beiging, in VSG responses.

Genes with reduced accessibility in VSG are associated with metabolic dysfunction and insulin resistance. Fkbp5 is highly upregulated by glucocorticoid stress-response hormones in adipose tissue and is implicated as a critical gene in glucocorticoid-induced insulin resistance. Its increased accessibility in adipocytes from SHAM-treated mice is consistent with the genomewide increase in accessibility of GR binding sites in the genome of those animals. FKBP5 is also upregulated in humans with type 2 diabetes. Notably, a polymorphism in FKBP5 in humans is associated with variations in weight loss outcomes after bariatric surgery (Hartmann et al., 2016). While the proteinase inhibitor Serpine1 (also called PAI-1) is predominantly expressed in visceral depots, obesity increases the subcutaneous expression of Serpine1. High levels of plasma PAl-1 are predictive of insulin resistance, type 2 diabetes, and hepatic steatosis. That Serpine1 accessibility is higher in SHAM adipocytes is also consistent with its being a VSG target that confers benefits. In opposition to the other genes with reduced accessibility in VSG, Lpin1 is not associated with metabolic dysfunction or insulin resistance. Instead, it is involved in the storage of lipids as triglycerides.

The most significantly differentially accessible gene in our dataset, with reduced accessibility in VSG adipocytes, was Scd1, a desaturase that converts saturated fatty acids (SFAs) into 
monounsaturated fatty acids (MUFAs). Notably, deletion of the Scd1 gene in mice leads to a wide array of changes in adiposity, energy storage and metabolism, and glucose homeostasis, each of which is highly relevant to responses to VSG (Cohen et al., 2002; Dobrzyn et al., 2004; Lee et al., 2004; Miyazaki et al., 2004, 2007, 2009) (X. Liu et al., 2009; Miyazaki et al., 2007). Overexpression of this gene is associated with obesity in humans in addition to its effect on insulin sensitivity (Sjögren et al., 2008). The functional importance of this accessibility change following VSG is described further below. Overall, the observed differentially accessible genes in VSG indicate a shift towards increased insulin sensitivity and thermogenic potential, both of which are among the benefits of VSG. Accordingly, our findings reveal molecular mechanisms underlying these benefits.

Because our data identified distinct mature adipocyte types, we wondered if the gene accessibility patterns described above for VSG and SHAM animals were present in specific adipocyte types. We found that beige-like AD1 harbored the observed accessibility changes for Pik3r1, Pparg, and Adcy5, while AD2 harbored the accessibility changes for Scd1, Adipor2, Adrb3, whereas there were fewer differences in gene accessibility of AD4 (fig 5c). This finding suggests that the AD4 subtype has less plasticity in responses to metabolic changes. Surprisingly, the white-like AD2, as opposed to beige-like AD1, had the most significant increase in beige-associated Adrb3 accessibility in VSG mice. This suggests that regardless of the treatment group, beige-like AD1 cells can respond to $\beta 3$-adrenergic receptor activation; however, white-like AD2 cells can acquire it after VSG. AD1 and AD2 also exhibited the most significant change in Scd1 of the subclusters.

In summary, ING VSG adipocytes have increased accessibility at genes associated with thermogenesis and beiging. VSG also leads to decreased accessibility in genes related to insulin resistance and type 2 diabetes, such as Scd1, Serpine1, and Fkbp5, and these changes are found in specific adipocyte subtypes. 
A
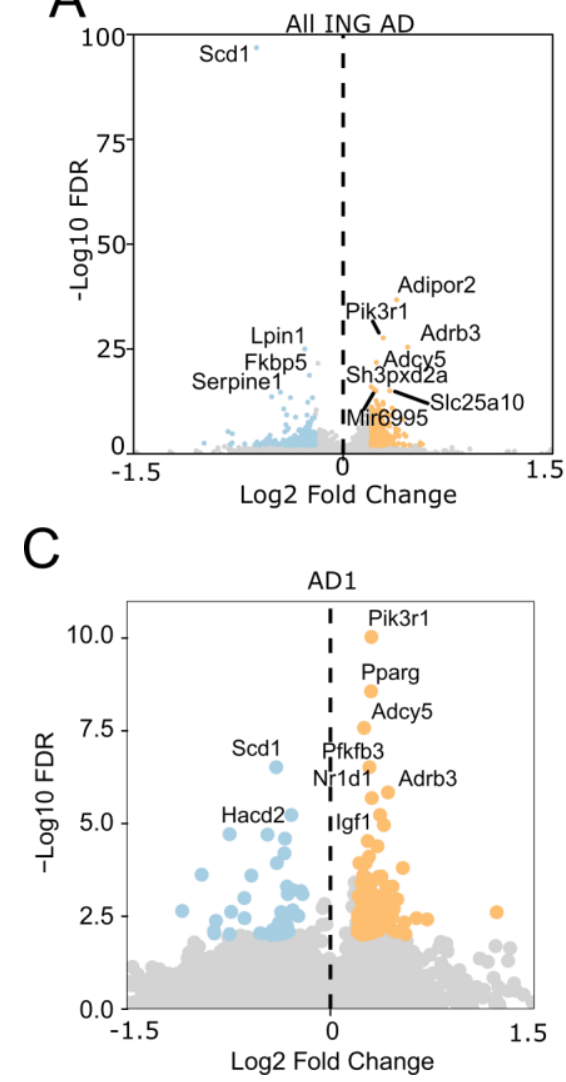

B
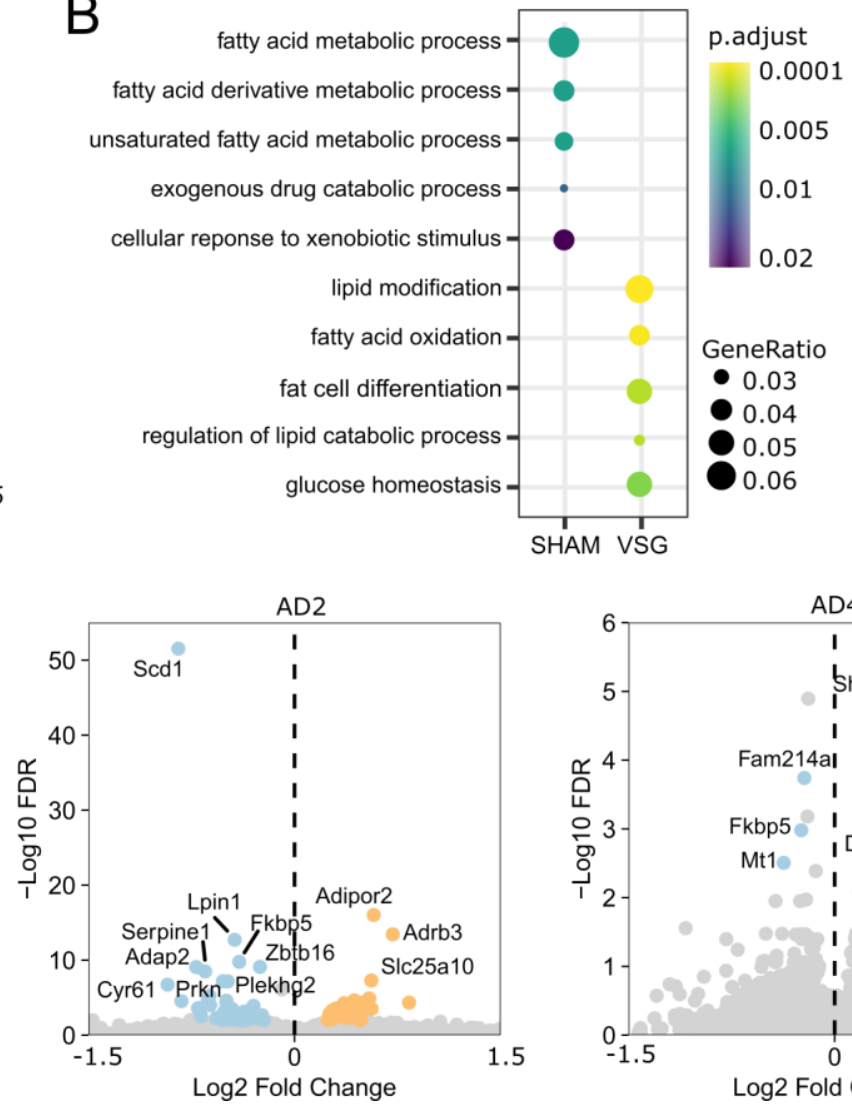

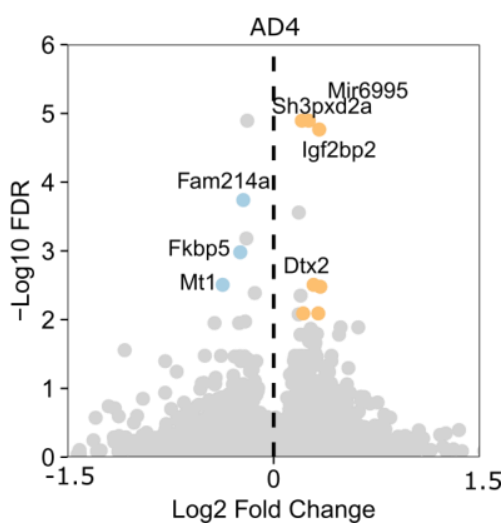

Figure 5. Accessibility changes in genes after VSG in inguinal mature adipocytes.

(A) Volcano plot of differentially accessible genes comparing VSG to SHAM ING adipocytes. Positive fold change indicates increased accessibility in VSG cells.

(B) Biological process gene ontology enrichment for the top 200 most significantly differentially accessible genes.

(C) Volcano plots of differentially accessible genes in VSG vs SHAM in each mature adipocyte cluster.

\section{Reduced SCD-1 accessibility decreases the accumulation of unsaturated fatty acids in the ING depot}

Our data identified genes enriched with open chromatin for each of the four types of samples analyzed, including both depots and both treatment groups (fig S4d). However, the most significant change in our dataset is Scd1, which is more accessible in AD2 adipocytes in ING SHAM (fig 5c). SCD1 converts saturated fatty acids, stearate and palmitate, into monounsaturated fatty acids, oleate and palmitoleate, respectively. Scd1 inhibition leads to an accumulation of stearate but not palmitate; this is partly due to the upregulation of fatty acid elongase 6 (Elov/6), which converts palmitate to stearate (Ralston et al., 2014). To validate whether these gene accessibility changes in ING after VSG have functional consequences on 
fatty acid profiles, we used mass spectrometry after one-step lipid extraction and methylation on the same adipose tissues used for our snATAC libraries. This method characterized the fatty acid chain length and position of double bonds and their quantitative levels (fig $6 \mathrm{~b}$ ). If reductions in Scd1 accessibility in ING VSG mice have functional consequences, we predicted there to be elevated levels of SFAs or reduced levels of MUFAs, leading to a lower overall ratio of MUFAs to SFAs in the ING depot of VSG mice. We further predicted that in EPI samples, which showed no accessibility changes at Scd1, there would be no significant alterations in the levels or ratios of lipid species between the treatment groups. These are precisely what we observed, demonstrating the functional consequences of Scd1 accessibility changes in the ING depot arising as a result of VSG (fig 6c-f). The desaturation index, which reports the ratio of MUFA to SFA for a fatty acid of a given chain length, was increased in the VSG ING depot by the accumulation of 16:0, and a reduction in 18:1n-9 lipids. However, these effects may be driven by the higher abundances of $16: 1 \mathrm{n}-7$ and 18:0, relative to the saturated and unsaturated forms of 16- and 18-carbon fatty acids, respectively. Importantly, in humans, the reductions in the desaturation index of 18-carbon fatty acids are positively correlated with insulin resistance (Sjögren et al., 2008). These results indicate that the regulation of Scd1 plays a vital role in the metabolic benefits of VSG through alterations in fatty acid modifications leading to improved insulin sensitivity (fig 6g). 


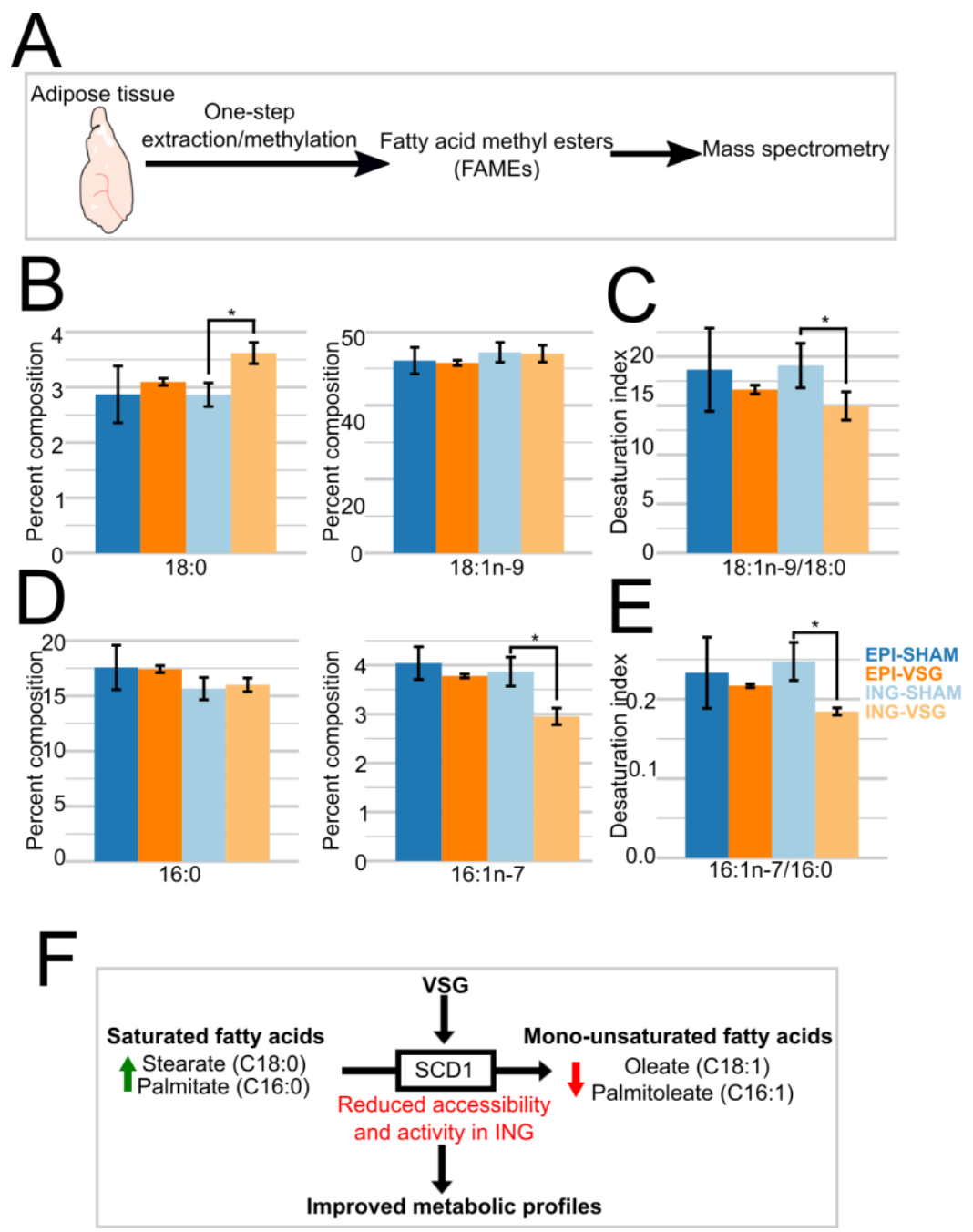

Figure 6. Reduced accessibility in Scd1 leads to less accumulation of unsaturated fatty acids

(A) Experimental design of determining FAME profiles, using three samples per depot and treatment group.

(B) 18-carbon fatty acid profiles, and (C) corresponding desaturation index.

(D) 16-carbon fatty acid profiles, and (E) corresponding desaturation index.

${ }^{*} \mathrm{p}<0.05$. Two-tailed students $t$-test was performed for (C,D,E,F) comparing VSG to SHAM within ING or EPI adipose samples.

(F) Model for the contributions of VSG to metabolic health through functional accessibility changes at Scd1. 


\section{Discussion}

We applied snATAC-seq profiling to mouse adipose tissues from weight-matched mice fed a high-fat diet for eight weeks before VSG or sham surgery, followed by tissue collection ten weeks after surgery (fig. 1a). Using this approach, we characterized the cellular composition of adipose, uncovered differences between visceral and subcutaneous depots, revealed functionally relevant effects of VSG upon each of these variables, including impacts on fatty acid metabolism, and identified regulatory motifs that are associated with these biological differences. These findings revealed developmentally- and environmentally influenced functional physiologic responses of adipose tissues. Because VSG imparts lasting changes that attenuate pathologies associated with obesity and diabetes, these findings also provide insights into functional pathways relevant to metabolic health, disease states, and the mechanisms underlying the benefits of bariatric surgical treatments for obesity.

Several challenges arise in the application of single-cell methods to whole mature adipose tissues. Mature adipocytes are large, lipid-filled, and buoyant in aqueous solutions, making droplet-based approaches difficult for their analyses. While the stromal vascular fraction of adipose does not have this issue, it includes only a portion of cells present in adipose and cannot reveal the depot- and VSG-specific changes in mature adipocytes we report. Nuclei extraction methods that worked well in our hands for many tissues from mice and humans led to clumping and poor yields with adipose. Optimizations described in the methods section circumvented these issues.

Our analyses identified four classes of mature adipocytes (AD1, 2, 3, 4), which collectively were the most abundant category of cells in adipose; we also identified adipocyte progenitors, endothelial cells, T-cells, and macrophages (fig 2a). In sham-operated mice, EPI and ING depots exhibited significant differences in the distributions of the mature adipocytes. AD1 was significantly more abundant (more than a 6-fold increase) in ING and had elevated accessibility relative to other adipocyte classes at Prdm16, a transcriptional cofactor that upregulates thermogenic genes. In contrast, AD4 was significantly more abundant in EPI (2-fold increase) and had elevated accessibility relative to other adipocyte classes at Npr3, a negative regulator of thermogenic genes (fig. 3a, (Bordicchia et al., 2012)). These findings indicate that AD1 are thermogenic beige-like adipocytes, AD4 are white adipocytes, and that these cellular differences, in part, account for the higher beiging potential in ING vs. EPI depots. We found further evidence for the higher beiging potential of ING depots: Within the AD4 adipocytes, those from ING depots had higher accessibility at Mir196a, which positively regulates beiging through suppression of Hox family genes. Interestingly, Hoxc10, a target of Mir196a and a negative regulator of beiging, is only expressed in subcutaneous adipose, like ING depots, but not in visceral, like EPI, or brown adipose. Additionally, Hoxc10 had higher accessibility in the AD4 population in ING relative to EPI. This indicated that AD4 adipocytes in ING depots have plasticity absent in EPI depots, enabling AD4 in ING to control the extent of beiging through positive and negative regulation. Furthermore, signaling triggers, such as those provided by VSG (Mori et al., 2012; Ng et al., 2017), can direct this plasticity in ING AD4 cells, leading to beiging.

In addition to these depot-specific differences in adipocyte abundances and chromatin states, the next most abundant cell type, APCs, were present in larger numbers in EPI than in ING. Genes involved in the regulation of cell fate, Wt1, and Tbx15, were also differentially accessible in APCs from the two depots, consistent with reports of the disparate developmental origins of these two depots (Chau et al., 2014; Sanchez-Gurmaches et al., 2015). These depot-specific APC differences may account for the differences seen in mature adipocytes derived from them in the two depots. Specifically, the increased accessibility in ING APCs at $T b x 15$, which is 
required for the beiging of white adipose tissue and increased thermogenesis (Ejarque et al., 2019; Sun et al., 2019), may enable APC-derived mature adipocytes in the ING depot to undergo beiging in response to VSG. In addition, regulators of macrophage state were differentially accessible in APCs from the two depots. EPI APCs showed higher accessibility at Tcf21, which has been linked to elevated IL-6 production by APCs in visceral adipose, which can induce M1-type pro-inflammatory macrophages (Akama \& Chun, 2018). In contrast, ING APCs showed higher accessibility at $C x c / 14$, which has been implicated in beiging, thermogenesis and induces M2-type anti-inflammatory macrophages (Cereijo et al., 2018). These differences in APCs between the two depots can, in part, account for the health hazards associated with accumulations of visceral as opposed to subcutaneous adipose.

There were significantly more macrophages and APCs in EPI than in ING, but we detected no abundance differences for endothelial cells or T-cells or depot-specific accessibility differences for these cell types. Failure to detect accessibility differences may be due to their lower cell abundances relative to adipocytes and APCs.

Subcutaneous and visceral adipose depots are known to respond differently to bariatric surgery in humans. Reductions in adiposity occur first in subcutaneous, followed by visceral depots (Galanakis et al., 2015; Toro-Ramos et al., 2015; Yoon et al., 2007). Consistent with human clinical findings, VSG induced different responses in ING vs. EPI depots in mice. We found many accessibility changes in ING in response to VSG two and a half months after surgery and fewer effects in EPI; most changes affected adipocytes. The most significant and highest magnitude changes to chromatin accessibility were in ING AD1 and AD2 adipocytes, with VSG increasing accessibility at genes affecting insulin sensitivity, lipid metabolism, and thermogenesis. In EPI adipocytes, VSG also increased accessibility at Pfkfb3, which increases insulin sensitivity. These findings identify changes in gene regulation that can contribute to the health benefits associated with VSG, including improvements in energy expenditure and glucose control. There were no significant changes within the APC population in response to VSG.

Scd1 encodes a fatty acid desaturase that catalyzes the rate-limiting step in the production of MUFAs from SFAs; it was the gene most significantly affected by VSG, showing reduced accessibility in AD1 and AD2 adipocytes after VSG. Accompanying this reduced Scd1 accessibility were reductions in the accumulation of MUFAs, or increased accumulation of SFAs in inguinal adipose from VSG mice, leading to reductions in the desaturation index of lipids present in the ING depot. Independent lines of evidence from in vivo and in vitro studies demonstrate that reduced SCD1 activity, and SFAs improve glucose and energy metabolism, adiposity, insulin responses, and adipose inflammation associated with obesity. Each of these is consistent with our findings implicating the importance of reduced Scd1 accessibility in mediating the beneficial effects of VSG. Genetic studies in mice have validated the metabolic benefits of reduced Scd1 expression in vivo for obesity- and diabetes-related traits. Mice bearing Scd1-inactivating mutations have lower fat mass, increased insulin sensitivity and signaling, lower white adipose inflammation, and higher metabolic rates than their wildtype counterparts (X. Liu et al., 2009; Miyazaki et al., 2004; Ntambi et al., 2002; Rahman et al., 2003). These effects are more dramatic in ob/ob leptin-deficient animals, where Scd1 deficiency also reduces body mass and liver triglycerides (Cohen et al., 2002), and improves insulin sensitivity (Miyazaki et al., 2009). SCD1 loss has also been associated with increased thermogenesis (Lee et al., 2004), fatty acid $\beta$-oxidation in muscles (Dobrzyn et al., 2004), and protection from carbohydrate-induced obesity (Miyazaki et al., 2007). These effects are likely due to the reduced fatty acid desaturation index that accompanies Scd1-inactivation, and not due to some secondary effect of SCD1 that is unrelated to its production of MUFAs. In vivo evidence comes from studies demonstrating that in Scd1-deficient mice, dietary oleate partially 
compensated for the hypothermia and elevated plasma glucose and liver glycogen stores (Lee et al., 2004).

Our findings identified cell- and gene-focused mechanisms that distinguish adipocyte responses to VSG. By extending our analyses to accessibility changes at intergenic regions, we were also able to identify gene regulatory mechanisms that may be responsible for these differences. STAT family transcription factor binding motifs were significantly enriched at regions of increased accessibility in adipocytes from VSG-treated mice. These factors are expressed in adipocytes, and in the case of STAT5a, regulates genes also found to exhibit increased accessibility in VSG, including those involved in fatty acid elongation, $\beta$-oxidation, and thermogenesis. In VSG mice, there were regions with reduced STAT5a accessibility, and these were proximal to genes with pro-inflammatory processes. These findings identify STAT5a as central to the beneficial effects of VSG and highlight that its binding is likely subject to regulatory cofactors that are important components of VSG responsiveness. We also identified motifs enriched at sites with reduced accessibility in adipocytes after VSG. These include the glucocorticoid receptor, NR1C3, which is associated with stress responses, impaired glucose metabolism, and obesity. Inferences from 3T3-L1 ChIP data indicate that the reduced accessibility at these motifs is associated with reduced occupancy by NR1C3 (Yu et al., 2010). Importantly, glucocorticoids participate in the regulation of Scd1. Accordingly, the reduced accessibility of NR1C3 motifs after VSG is likely to contribute to the beneficial changes in the fatty acid metabolism we observed. Collectively, these motif findings identify likely regulatory mechanisms that are central to the effects of VSG. 
bioRxiv preprint doi: https://doi org/10.1101/2021.06.22 449469; this version posted June 22, 2021. The copyright holder for this preprint (which was not certified by peer review) is the author/funder, who has granted bioRxiv a license to display the preprint in perpetuity. It is made available under aCC-BY-NC-ND 4.0 International license.

\section{Acknowledgements}

The Cornell University Biotechnology Resource Center (BRC) for genomic sequencing. Chris Donahue for nuclei FACS. Seoyeon Lee from the Soloway lab at Cornell University for helpful discussions. Anne McGavigan and coauthors from the Cummings lab at Cornell University for use of adipose tissues. 


\section{References}

Akama, T., \& Chun, T. H. (2018). Transcription factor 21 (TCF21) promotes proinflammatory interleukin 6 expression and extracellular matrix remodeling in visceral adipose stem cells. Journal of Biological Chemistry, 293(17), 6603-6610. https://doi.org/10.1074/jbc.RA117.000456

Bordicchia, M., Liu, D., Amri, E. Z., Ailhaud, G., Dessì-Fulgheri, P., Zhang, C., Takahashi, N., Sarzani, R., \& Collins, S. (2012). Cardiac natriuretic peptides act via p38 MAPK to induce the brown fat thermogenic program in mouse and human adipocytes. Journal of Clinical Investigation, 122(3), 1022-1036. https://doi.org/10.1172/JCI59701

Buchwald, H., Avidor, Y., Braunwald, E., Jensen, M. D., Pories, W., Fahrbach, K., \& Schoelles, K. (2004). Bariatric Surgery. JAMA, 292(14), 1724. https://doi.org/10.1001/jama.292.14.1724

Cereijo, R., Gavaldà-Navarro, A., Cairó, M., Quesada-López, T., Villarroya, J., Morón-Ros, S., Sánchez-Infantes, D., Peyrou, M., Iglesias, R., Mampel, T., Turatsinze, J. V., Eizirik, D. L., Giralt, M., \& Villarroya, F. (2018). CXCL14, a Brown Adipokine that Mediates Brown-Fat-toMacrophage Communication in Thermogenic Adaptation. Cell Metabolism, 28(5), 750763.e6. https://doi.org/10.1016/j.cmet.2018.07.015

Chau, Y. Y., Bandiera, R., Serrels, A., Martínez-Estrada, O. M., Qing, W., Lee, M., Slight, J., Thornburn, A., Berry, R., Mchaffie, S., Stimson, R. H., Walker, B. R., Chapuli, R. M., Schedl, A., \& Hastie, N. (2014). Visceral and subcutaneous fat have different origins and evidence supports a mesothelial source. Nature Cell Biology, 16(4), 367-375. https://doi.org/10.1038/ncb2922

Chi, J., Wu, Z., Choi, C. H. J., Nguyen, L., Tegegne, S., Ackerman, S. E., Crane, A., Marchildon, F., Tessier-Lavigne, M., \& Cohen, P. (2018). Three-Dimensional Adipose Tissue Imaging Reveals Regional Variation in Beige Fat Biogenesis and PRDM16-Dependent Sympathetic Neurite Density. Cell Metabolism, 27(1), 226-236.e3. https://doi.org/10.1016/j.cmet.2017.12.011

Cho, D. S., Lee, B., \& Doles, J. D. (2019). Refining the adipose progenitor cell landscape in healthy and obese visceral adipose tissue using single-cell gene expression profiling. Life Science Alliance, 2(6). https://doi.org/10.26508/lsa.201900561

Cohen, P., Levy, J. D., Zhang, Y., Frontini, A., Kolodin, D. P., Svensson, K. J., Lo, J. C., Zeng, X., Ye, L., Khandekar, M. J., Wu, J., Gunawardana, S. C., Banks, A. S., Camporez, J. P. G., Jurczak, M. J., Kajimura, S., Piston, D. W., Mathis, D., Cinti, S., ... Spiegelman, B. M. (2014). Ablation of PRDM16 and beige adipose causes metabolic dysfunction and a subcutaneous to visceral fat switch. Cell, 156(1-2), 304-316. https://doi.org/10.1016/j.cell.2013.12.021

Cohen, P., Miyazaki, M., Socci, N. D., Hagge-Greenberg, A., Liedtke, W., Soukas, A. A., Sharma, R., Hudgins, L. C., Ntambi, J. M., \& Friedman, J. M. (2002). Role for stearoyl-CoA desaturase-1 in leptin-mediated weight loss. Science, 297(5579), 240-243. https://doi.org/10.1126/science. 1071527

Coulter, A. A., \& Stephens, J. M. (2006). STAT5 activators modulate acyl CoA oxidase (AOX) expression in adipocytes and STAT5A binds to the AOX promoter in vitro. Biochemical and Biophysical Research Communications, 344(4), 1342-1345.

https://doi.org/10.1016/j.bbrc.2006.04.071 
Cummings, B. P., Bettaieb, A., Graham, J. L., Stanhope, K. L., Kowala, M., Haj, F. G., Chouinard, M. L., \& Havel, P. J. (2012). Vertical sleeve gastrectomy improves glucose and lipid metabolism and delays diabetes onset in UCD-T2DM rats. Endocrinology, 153(8), 3620-3632. https://doi.org/10.1210/en.2012-1131

Dobrzyn, P., Dobrzyn, A., Miyazaki, M., Cohen, P., Asilmaz, E., Hardie, D. G., Friedman, J. M., \& Ntambi, J. M. (2004). Stearoyl-CoA desaturase 1 deficiency increases fatty acid oxidation by activating AMP-activated protein kinase in liver. Proceedings of the National Academy of Sciences of the United States of America, 101(17), 6409-6414. https://doi.org/10.1073/pnas.0401627101

Ejarque, M., Ceperuelo-Mallafré, V., Serena, C., Maymo-Masip, E., Duran, X., Díaz-Ramos, A., Millan-Scheiding, M., Núñez-Álvarez, Y., Núñez-Roa, C., Gama, P., Garcia-Roves, P. M., Peinado, M. A., Gimble, J. M., Zorzano, A., Vendrell, J., \& Fernández-Veledo, S. (2019). Adipose tissue mitochondrial dysfunction in human obesity is linked to a specific DNA methylation signature in adipose-derived stem cells. International Journal of Obesity, 43(6), 1256-1268. https://doi.org/10.1038/s41366-018-0219-6

Galanakis, C. G., Daskalakis, M., Manios, A., Xyda, A., Karantanas, A. H., \& Melissas, J. (2015). Computed tomography-based assessment of abdominal adiposity changes and their impact on metabolic alterations following bariatric surgery. World Journal of Surgery, 39(2), 417-423. https://doi.org/10.1007/s00268-014-2826-2

Garcés, R., \& Mancha, M. (1993). One-step lipid extraction and fatty acid methyl esters preparation from fresh plant tissues. Analytical Biochemistry, 211(1), 139-143. https://doi.org/10.1006/abio.1993.1244

Garibay, D., Lou, J., Lee, S. A., Zaborska, K. E., Weissman, M. H., Sloma, E., Donahue, L., Miller, A. D., White, A. C., Michael, M. D., Sloop, K. W., \& Cummings, B. P. (2018). $\beta$ Cell GLP-1R Signaling Alters a Cell Proglucagon Processing after Vertical Sleeve Gastrectomy in Mice. Cell Reports, 23(4), 967-973. https://doi.org/10.1016/j.celrep.2018.03.120

Gealekman, O., Guseva, N., Hartigan, C., Apotheker, S., Gorgoglione, M., Gurav, K., Tran, K. Van, Straubhaar, J., Nicoloro, S., Czech, M. P., Thompson, M., Perugini, R. A., \& Corvera, S. (2011). Depot-specific differences and insufficient subcutaneous adipose tissue angiogenesis in human obesity. Circulation, 123(2), 186-194. https://doi.org/10.1161/CIRCULATIONAHA.110.970145

Gleeson, P. A. (2014). The role of endosomes in innate and adaptive immunity. In Seminars in Cell and Developmental Biology (Vol. 31, pp. 64-72). Elsevier Ltd. https://doi.org/10.1016/j.semcdb.2014.03.002

Granja, J. M., Corces, M. R., Pierce, S. E., Bagdatli, S. T., Choudhry, H., Chang, H. Y., \& Greenleaf, W. J. (2021). ArchR is a scalable software package for integrative single-cell chromatin accessibility analysis. Nature Genetics, 53(3), 403-411. https://doi.org/10.1038/s41588-021-00790-6

Harris, D. A., Mina, A., Cabarkapa, D., Heshmati, K., Subramaniam, R., Banks, A. S., Tavakkoli, A., \& Sheu, E. G. (2020). Sleeve gastrectomy enhances glucose utilization and remodels adipose tissue independent of weight loss. American Journal of Physiology - Endocrinology and Metabolism, 318(5), E678-E688. https://doi.org/10.1152/ajpendo.00441.2019

Hartmann, I. B., Fries, G. R., Bücker, J., Scotton, E., von Diemen, L., \& Kauer-Sant'Anna, M. (2016). The FKBP5 polymorphism rs 1360780 is associated with lower weight loss after 
bariatric surgery: 26 months of follow-up. Surgery for Obesity and Related Diseases, 12(8), 1554-1560. https://doi.org/10.1016/j.soard.2016.04.016

Ikeda, K., Kang, Q., Yoneshiro, T., Camporez, J. P., Maki, H., Homma, M., Shinoda, K., Chen, Y., Lu, X., Maretich, P., Tajima, K., Ajuwon, K. M., Soga, T., \& Kajimura, S. (2017). UCP1independent signaling involving SERCA2bmediated calcium cycling regulates beige fat thermogenesis and systemic glucose homeostasis. Nature Medicine, 23(12), 1454-1465. https://doi.org/10.1038/nm.4429

Jahansouz, C., Xu, H., Hertzel, A. V., Kizy, S., Steen, K. A., Foncea, R., Serrot, F. J., Kvalheim, N., Luthra, G., Ewing, K., Leslie, D. B., Ikramuddin, S., \& Bernlohr, D. A. (2018).

Partitioning of adipose lipid metabolism by altered expression and function of PPAR isoforms after bariatric surgery. International Journal of Obesity, 42(2), 139-146. https://doi.org/10.1038/ijo.2017.197

Jiang, Y., Berry, D. C., \& Graff, J. M. (2017). Distinct cellular and molecular mechanisms for $\beta 3$ adrenergic receptor-induced beige adipocyte formation. ELife, 6. https://doi.org/10.7554/eLife.30329

Kelly, A. S., Ryder, J. R., Marlatt, K. L., Rudser, K. D., Jenkins, T., \& Inge, T. H. (2016). Changes in inflammation, oxidative stress and adipokines following bariatric surgery among adolescents with severe obesity. International Journal of Obesity, 40(2), 275-280. https://doi.org/10.1038/ijo.2015.174

Kwok, K. H. M., Lam, K. S. L., \& Xu, A. (2016). Heterogeneity of white adipose tissue: molecular basis and clinical implications. Experimental \& Molecular Medicine 2016 48:3, 48(3), e215e215. https://doi.org/10.1038/emm.2016.5

Lee, S. H., Dobrzyn, A., Dobrzyn, P., Rahman, S. M., Miyazaki, M., \& Ntambi, J. M. (2004). Lack of stearoyl-CoA desaturase 1 upregulates basal thermogenesis but causes hypothermia in a cold environment. Journal of Lipid Research, 45(9), 1674-1682. https://doi.org/10.1194/jlr.M400039-JLR200

Liu, T., Mi, L., Xiong, J., Orchard, P., Yu, Q., Yu, L., Zhao, X. Y., Meng, Z. X., Parker, S. C. J., Lin, J. D., \& Li, S. (2020). BAF60a deficiency uncouples chromatin accessibility and cold sensitivity from white fat browning. Nature Communications, 11(1), 1-15. https://doi.org/10.1038/s41467-020-16148-1

Liu, W., Ji, Y., Zhang, B., Chu, H., Yin, C., \& Xiao, Y. (2020). Stat5a promotes brown adipocyte differentiation and thermogenic program through binding and transactivating the Kdm6a promoter. Cell Cycle, 19(8), 895-905. https://doi.org/10.1080/15384101.2020.1731644

Liu, X., Miyazaki, M., Flowers, M. T., Sampath, H., Zhao, M., Chu, K., Paton, C. M., Diane, ;, Joo, S., \& Ntambi, J. M. (2009). Loss of Stearoyl-CoA Desaturase-1 Attenuates Adipocyte Inflammation Effects of Adipocyte-Derived Oleate. https://doi.org/10.1161/ATVBAHA.109.195636

McGavigan, A. K., Garibay, D., Henseler, Z. M., Chen, J., Bettaieb, A., Haj, F. G., Ley, R. E., Chouinard, M. L., \& Cummings, B. P. (2017). TGR5 contributes to glucoregulatory improvements after vertical sleeve gastrectomy in mice. Gut, 66(2), 226-234. https://doi.org/10.1136/gutjnl-2015-309871

McGavigan, A. K., Henseler, Z. M., Garibay, D., Butler, S. D., Jayasinghe, S., Ley, R. E., Davisson, R. L., \& Cummings, B. P. (2017). Vertical sleeve gastrectomy reduces blood pressure and hypothalamic endoplasmic reticulum stress in mice. DMM Disease Models 
and Mechanisms, 10(3), 235-243. https://doi.org/10.1242/dmm.027474

Milani, P., Escalante-Chong, R., Shelley, B. C., Patel-Murray, N. L., Xin, X., Adam, M., Mandefro, B., Sareen, D., Svendsen, C. N., \& Fraenkel, E. (2016). Cell freezing protocol suitable for ATAC-Seq on motor neurons derived from human induced pluripotent stem cells. Scientific Reports, 6(1), 1-10. https://doi.org/10.1038/srep25474

Miyazaki, M., Dobrzyn, A., Sampath, H., Lee, S. H., Weng, C. M., Chu, K., Peters, J. M., Gonzalez, F. J., \& Ntambi, J. M. (2004). Reduced adiposity and liver steatosis by stearoylCoA desaturase deficiency are independent of peroxisome proliferator-activated receptora. Journal of Biological Chemistry, 279(33), 35017-35024. https://doi.org/10.1074/jbc.M405327200

Miyazaki, M., Flowers, M. T., Sampath, H., Chu, K., Otzelberger, C., Liu, X., \& Ntambi, J. M. (2007). Hepatic Stearoyl-CoA Desaturase-1 Deficiency Protects Mice from CarbohydrateInduced Adiposity and Hepatic Steatosis. Cell Metabolism, 6(6), 484-496. https://doi.org/10.1016/j.cmet.2007.10.014

Miyazaki, M., Sampath, H., Liu, X., Flowers, M. T., Chu, K., Dobrzyn, A., \& Ntambi, J. M. (2009). Stearoyl-CoA desaturase-1 deficiency attenuates obesity and insulin resistance in leptinresistant obese mice. Biochemical and Biophysical Research Communications, 380(4), 818-822. https://doi.org/10.1016/j.bbrc.2009.01.183

Mori, M., Nakagami, H., Rodriguez-Araujo, G., Nimura, K., \& Kaneda, Y. (2012). Essential role for miR-196a in brown adipogenesis of white fat progenitor cells. PLoS Biology, 10(4), 1001314. https://doi.org/10.1371/journal.pbio.1001314

Ng, Y., Tan, S. X., Chia, S. Y., Tan, H. Y. A., Gun, S. Y., Sun, L., Hong, W., \& Han, W. (2017). HOXC10 suppresses browning of white adipose tissues. Experimental and Molecular Medicine, 49(2), 292. https://doi.org/10.1038/emm.2016.144

Ntambi, J. M., Miyazaki, M., Stoehr, J. P., Lan, H., Kendziorski, C. M., Yandell, B. S., Song, Y., Cohen, P., Friedman, J. M., \& Attie, A. D. (2002). Loss of stearoyl-CoA desaturase-1 function protects mice against adiposity. Proceedings of the National Academy of Sciences of the United States of America, 99(17), 11482-11486. https://doi.org/10.1073/pnas.132384699

Pories, W. J., Swanson, M. S., MacDonald, K. G., Long, S. B., Morris, P. G., Brown, B. M., Barakat, H. A., DeRamon, R. A., Israel, G., \& Dolezal, J. M. (1995). Who would have thought it? An operation proves to be the most effective therapy for adult-onset diabetes mellitus. Annals of Surgery, 222(3), 339-350; discussion 350-2. https://doi.org/10.1097/00000658-199509000-00011

Preissl, S., Fang, R., Huang, H., Zhao, Y., Raviram, R., Gorkin, D. U., Zhang, Y., Sos, B. C., Afzal, V., Dickel, D. E., Kuan, S., Visel, A., Pennacchio, L. A., Zhang, K., \& Ren, B. (2018). Single-nucleus analysis of accessible chromatin in developing mouse forebrain reveals cell-type-specific transcriptional regulation. Nature Neuroscience, 21(3), 432-439. https://doi.org/10.1038/s41593-018-0079-3

Rahman, S. M., Dobrzyn, A., Dobrzyn, P., Lee, S. H., Miyazaki, M., \& Ntambi, J. M. (2003). Stearoyl-CoA desaturase 1 deficiency elevates insulin-signaling components and downregulates protein-tyrosine phosphatase $1 \mathrm{~B}$ in muscle. Proceedings of the National Academy of Sciences of the United States of America, 100(19), 11110-11115. https://doi.org/10.1073/pnas.1934571100 
Rajakumari, S., Wu, J., Ishibashi, J., Lim, H. W., Giang, A. H., Won, K. J., Reed, R. R., \& Seale, P. (2013). EBF2 determines and maintains brown adipocyte identity. Cell Metabolism, 17(4), 562-574. https://doi.org/10.1016/j.cmet.2013.01.015

Ralston, J. C., Badoud, F., Cattrysse, B., McNicholas, P. D., \& Mutch, D. M. (2014). Inhibition of stearoyl-CoA desaturase-1 in differentiating 3T3-L1 preadipocytes upregulates elongase 6 and downregulates genes affecting triacylglycerol synthesis. International Journal of Obesity, 38(11), 1449-1456. https://doi.org/10.1038/ijo.2014.35

Rinaldi, V. D., Donnard, E., Gellatly, K. J., Rasmussen, M., Kucukural, A., Yukselen, O., Garber, M., Sharma, U., \& Rando, O. J. (2020). An atlas of cell types in the mouse epididymis and vas deferens. ELife, 9, 1-49. https://doi.org/10.7554/eLife.55474

Rosenwald, M., Perdikari, A., Rülicke, T., \& Wolfrum, C. (2013). Bi-directional interconversion of brite and white adipocytes. Nature Cell Biology, 15(6), 659-667.

https://doi.org/10.1038/ncb2740

Sanchez-Gurmaches, J., Hsiao, W. Y., \& Guertin, D. A. (2015). Highly selective in vivo labeling of subcutaneous white adipocyte precursors with Prx1-Cre. Stem Cell Reports, 4(4), 541550. https://doi.org/10.1016/j.stemcr.2015.02.008

Savu, M. K., Phillips, S. A., Oh, D. K., Park, K., Gerlan, C., Ciaraldi, T. P., \& Henry, R. R. (2009). Response of adiponectin and its receptors to changes in metabolic state after gastric bypass surgery: dissociation between adipose tissue expression and circulating levels. Surgery for Obesity and Related Diseases, 5(2), 172-180.

https://doi.org/10.1016/j.soard.2008.08.013

Schupp, J. C., Adams, T. S., Cosme, C., Sam, M., Raredon, B., Omote, N., De Frias, S. P., Rose, K.-A., Manning, E., Sauler, M., Deiuliis, G., Ahangari, F., Neumark, N., Yuan, Y., Habermann, A. C., Gutierrez, A. J., Bui, L. T., Meyer, K. B., Nawijn, M. C., ... Kaminski, N. (2020). Integrated Single Cell Atlas of Endothelial Cells of the Human Lung. BioRxiv, 2020.10.21.347914. https://doi.org/10.1101/2020.10.21.347914

Schweitzer, P., Harris, A., Mandelman, D., Jackson, S., Cifuentes, F., \& Degoricija, L. (2014). Precise Quantification of Next Generation Sequencing Ion Torrent ${ }^{\mathrm{TM}}$ and Illumina Libraries using the QuantStudio ${ }^{\mathrm{TM}}$ 3D Digital PCR Platform. Journal of Biomolecular Techniques : JBT, 25(Suppl), S15. /pmc/articles/PMC4162198/?report=abstract

Seale, P., Conroe, H. M., Estall, J., Kajimura, S., Frontini, A., Ishibashi, J., Cohen, P., Cinti, S., \& Spiegelman, B. M. (2011). Prdm16 determines the thermogenic program of subcutaneous white adipose tissue in mice. Journal of Clinical Investigation, 121(1), 96105. https://doi.org/10.1172/JCl44271

Sjögren, P., Sierra-Johnson, J., Gertow, K., Rosell, M., Vessby, B., De Faire, U., Hamsten, A., Hellenius, M. L., \& Fisher, R. M. (2008). Fatty acid desaturases in human adipose tissue: Relationships between gene expression, desaturation indexes and insulin resistance. Diabetologia, 51(2), 328-335. https://doi.org/10.1007/s00125-007-0876-9

Sowers, J. R. (2008). Endocrine Functions of Adipose Tissue: Focus on Adiponectin. Clinical Cornerstone, 9(1), 32-40. https://doi.org/10.1016/S1098-3597(08)60026-5

Spektor, R., Yang, J. W., Lee, S., \& Soloway, P. D. (2019). Single cell ATAC-seq identifies broad changes in neuronal abundance and chromatin accessibility in Down Syndrome. BioRxiv, 561191. https://doi.org/10.1101/561191 
Stine, R. R., Shapira, S. N., Lim, H. W., Ishibashi, J., Harms, M., Won, K. J., \& Seale, P. (2016). EBF2 promotes the recruitment of beige adipocytes in white adipose tissue. Molecular Metabolism, 5(1), 57-65. https://doi.org/10.1016/j.molmet.2015.11.001

Sun, W., Zhao, X., Wang, Z., Chu, Y., Mao, L., Lin, S., Gao, X., Song, Y., Hui, X., Jia, S., Tang, S., Xu, Y., Xu, A., Loomes, K., Wang, C., Wu, D., \& Nie, T. (2019). Tbx15 is required for adipocyte browning induced by adrenergic signaling pathway. Molecular Metabolism, 28, 48-57. https://doi.org/10.1016/j.molmet.2019.07.004

Toro-Ramos, T., Goodpaster, B. H., Janumala, I., Lin, S., Strain, G. W., Thornton, J. C., Kang, P., Courcoulas, A. P., Pomp, A., \& Gallagher, D. (2015). Continued Loss in Visceral and Intermuscular Adipose Tissue in Weight-Stable Women Following Bariatric Surgery. Obesity (Silver Spring, Md.), 23(1), 62. https://doi.org/10.1002/OBY.20932

Ukropec, J., Anunciado, R. P., Ravussin, Y., Hulver, M. W., \& Kozak, L. P. (2006). UCP1independent Thermogenesis in White Adipose Tissue of Cold-acclimated Ucp1-/- Mice. Journal of Biological Chemistry, 281(42), 31894-31908. https://doi.org/10.1016/s00219258(19)84104-2

Velazquez-Villegas, L. A., Perino, A., Lemos, V., Zietak, M., Nomura, M., Pols, T. W. H., \& Schoonjans, K. (2018). TGR5 signalling promotes mitochondrial fission and beige remodelling of white adipose tissue. Nature Communications, 9(1), 1-13. https://doi.org/10.1038/s41467-017-02068-0

Vijay, J., Gauthier, M. F., Biswell, R. L., Louiselle, D. A., Johnston, J. J., Cheung, W. A., Belden, B., Pramatarova, A., Biertho, L., Gibson, M., Simon, M. M., Djambazian, H., Staffa, A., Bourque, G., Laitinen, A., Nystedt, J., Vohl, M. C., Fraser, J. D., Pastinen, T., ... Grundberg, E. (2020). Single-cell analysis of human adipose tissue identifies depot- and disease-specific cell types. Nature Metabolism, 2(1), 97-109. https://doi.org/10.1038/s42255-019-0152-6

Wang, W., Kissig, M., Rajakumari, S., Huang, L., Lim, H. W., Won, K. J., \& Seale, P. (2014). Ebf2 is a selective marker of brown and beige adipogenic precursor cells. Proceedings of the National Academy of Sciences of the United States of America, 111(40), 14466-14471. https://doi.org/10.1073/pnas.1412685111

Wu, J., Boström, P., Sparks, L. M., Ye, L., Choi, J. H., Giang, A. H., Khandekar, M., Virtanen, K. A., Nuutila, P., Schaart, G., Huang, K., Tu, H., Van Marken Lichtenbelt, W. D., Hoeks, J., Enerbäck, S., Schrauwen, P., \& Spiegelman, B. M. (2012). Beige adipocytes are a distinct type of thermogenic fat cell in mouse and human. Cell, 150(2), 366-376. https://doi.org/10.1016/j.cell.2012.05.016

Yoon, D. Y., Kim, H. K., Kim, J. A., Choi, C. S., Yun, E. J., Chang, S. K., Lee, Y. J., \& Park, C. $\mathrm{H}$. (2007). Changes in the abdominal fat distribution after gastrectomy: Computed tomography assessment. ANZ Journal of Surgery, 77(3), 121-125. https://doi.org/10.1111/j.1445-2197.2006.03990.x

Yu, C. Y., Mayba, O., Lee, J. V., Tran, J., Harris, C., Speed, T. P., \& Wang, J. C. (2010). Genome-wide analysis of glucocorticoid receptor binding regions in adipocytes reveal gene network involved in triglyceride homeostasis. PLOS ONE, 5(12), 15188. https://doi.org/10.1371/journal.pone.0015188

Zhang, Y., Zhao, H., Cao, Z., Sun, X., Zhang, C., Cai, W., Liu, R., Hu, S., \& Qin, M. (2014). A Randomized Clinical Trial of Laparoscopic Roux-en-Y Gastric Bypass and Sleeve 
bioRxiv preprint doi: https://doi org/10.1101/2021.06.22 449469; this version posted June 22, 2021. The copyright holder for this preprint (which was not certified by peer review) is the author/funder, who has granted bioRxiv a license to display the preprint in perpetuity. It is made available under aCC-BY-NC-ND 4.0 International license.

Gastrectomy for the Treatment of Morbid Obesity in China: a 5-Year Outcome. Obesity Surgery, 24(10), 1617-1624. https://doi.org/10.1007/s11695-014-1258-2

Zhao, P., \& Stephens, J. M. (2013). Identification of STAT target genes in adipocytes. JAKSTAT, 2(2), e23092. https://doi.org/10.4161/jkst.23092 


\section{Methods}

\section{Mice}

At 2 months of age, C57/BL/6J mice were placed on a $60 \%$ energy from fat high-fat diet (HFD) for eight weeks then underwent VSG, or sham surgery as described in McGavigan et al. mice were maintained on HFD throughout the study. Mice were euthanized at 2.5 months after surgery, and the inguinal (ING) and epididymal (EPI) adipose depots were dissected without removing lymph nodes, frozen, and stored.

\section{Nuclei isolation}

Whole adipose depots were finely minced on dry ice. Then $80 \mathrm{mg}$ of adipose tissue was transferred to a pre-chilled Dounce homogenizer (Wheaton \#357546) with $30 \mathrm{~mL}$ of HB (320 $\mathrm{mM}$ sucrose, $0.1 \mathrm{mM}$ EDTA, 0.1\% NP40, $5 \mathrm{mM} \mathrm{CaCl}$, $3 \mathrm{mM} \mathrm{Mg}(\mathrm{Ac}) 2,10 \mathrm{mM}$ Tris $\mathrm{pH} 7.4$, protease inhibitors (Pierce \#88666), $0.016 \mathrm{mM} \mathrm{PMSF).} \mathrm{All} \mathrm{steps} \mathrm{were} \mathrm{performed} \mathrm{on} \mathrm{ice} \mathrm{or} \mathrm{at}$ $4^{\circ} \mathrm{C}$. The tissue was first homogenized with 10-15 strokes using a loose pestle then an additional 10 strokes with the tight pestle. The homogenized tissue was then filtered through a $70 \mu \mathrm{m}$ cell strainer (BD Biosciences \#352350) and centrifuged for 5 mins at $500 \mathrm{~g}$ in a centrifuge with a swinging bucket rotor. The supernatant was removed, the cell pellet washed with ATACRSB (10 mM Tris-HCL pH 7.4, $10 \mathrm{mM} \mathrm{NaCl}, 3 \mathrm{mM} \mathrm{MgCl} 2, \mathrm{H} 2 \mathrm{O}$ ), then centrifuged for 10 minutes at $750 \mathrm{~g}$. After removing the supernatant, nuclei were resuspended in $1 \mathrm{~mL}$ of OMNIATAC buffer (10 mM Tris pH7.4, $5 \mathrm{mM} \mathrm{MgCl}$, 10\% DMF, 33\% 1x PBS(no Ca++ or Mg++), 0.1\% Tween-20, 0.01\% Digitonin (ThermoFisher \#BN2006)), counted on a hemacytometer, and the concentration was adjusted to 100,000 nuclei $/ \mathrm{mL}$.

\section{Library preparation}

The snATAC library preparation protocol was based on Preissl et al. and Spektor et al. with some modifications (Preissl et al., 2018; Spektor et al., 2019). For tagmentation with Tn5 transposase, $8 \mu \mathrm{L}$ of the nuclei suspension was added to each well of a 96 well plate. Then, 1 $\mu \mathrm{L}$ of ME-C barcoded transposome loaded Tn5 and $1 \mu \mathrm{L}$ of ME-D barcoded transposome loaded Tn5 were added to each well. Tagmentation was carried out at $50^{\circ} \mathrm{C}$ for $30 \mathrm{mins}$. $10 \mu \mathrm{L}$ of $40 \mu \mathrm{M}$ EDTA was added to each well, gently vortexed, and incubated for 15 minutes at $37^{\circ} \mathrm{C}$ to inactivate Tn5 transposase. $20 \mu \mathrm{L}$ of sorting buffer (2\% BSA, 2mM EDTA in PBS (no Ca++ or $\mathrm{Mg++})$ ) was added to each well, and all wells were combined. For the freeze-thaw test, nuclei were centrifuged for 5 mins at $500 \mathrm{~g}$ and resuspended in $500 \mu \mathrm{L}$ of freeze buffer $(10 \%$ DMSO, $90 \%$ sort buffer) and slowly frozen and stored at $-80^{\circ} \mathrm{C}$.

Nuclei were stained with Draq7(1:100; Abcam \#ab109202), filtered through a $30 \mu \mathrm{m}$ filcon cell strainer (BD Biosciences \#340597), and 25 nuclei were sorted into each well of a 96 well plate containing $16.5 \mu \mathrm{L}$ of sort EB (10mM Tris $\mathrm{pH} 8,2 \% \mathrm{BSA}, \mathrm{H} 2 \mathrm{O})$ per well using a BD FACSAria Fusion. For PCR amplification, $2 \mu \mathrm{L}$ of $0.2 \%$ SDS was added to each well and incubated for 7 minutes at $55^{\circ} \mathrm{C}$. Next, $2.5 \mu \mathrm{L}$ of $10 \%$ Triton X-100, $2 \mu \mathrm{L}$ of $25 \mu \mathrm{M}$ Primer i5, $2 \mu \mathrm{L}$ of $25 \mu \mathrm{M}$ Primer i7, and $25 \mu \mathrm{L}$ of $2 X$ PCR mix with Q5 DNA polymerase (NEB \#M0491S) (Q5 5X buffer, dNTP, Q5 enzyme, GC enhancer, H2O) was added to each well. All PCR reactions were run in the same thermocycler with the following program: $72^{\circ} \mathrm{C} 5$ minutes, $98^{\circ} \mathrm{C} 30$ seconds, 15 cycles of $\left(98^{\circ} \mathrm{C} 10\right.$ seconds, $63^{\circ} \mathrm{C} 30$ seconds, $72^{\circ} \mathrm{C} 1$ minute), and $72^{\circ} \mathrm{C} 5$ minutes. After PCR, all wells were pooled, then $24 \mathrm{~mL}$ buffer PB (5:1, Qiagen \#19066) and 1.2mL NaOAc were added sequentially. Samples were each run through a separate MinElute column (Qiagen \#28004) 
using a QIAvac apparatus (Qiagen \#19413), then washed twice with $750 \mathrm{~mL}$ Buffer PE (Qiagen \#19065). The sample was then eluted in $40 \mathrm{uL}$ of Buffer EB (10 mM Tris pH 8) and centrifuged at max speed on a tabletop centrifuge for 2 minutes. The following size selection steps were performed at room temperature. First, large fragments were removed with $0.5 \mathrm{X}$ vol AMPure XP (Beckman Coulter \#A63880) purification, and then size selected 3 times with 1.5X vol AMPure XP consecutively to remove PCR primers according to the manufacturer's protocol.

\section{Library quality control and sequencing}

Each library was measured using a bioanalyzer for the expected nucleosomal patterning. Libraries were pooled using digital PCR on a Bio-Rad QX200 droplet digital PCR system using the following oligos: forward, AGCAGAAGACGGCATACGAGAT; reverse ATACGGCGACCACCGAGATC; internal /56-FAM/TCTTATACACATCTGAGGCGG/3BHQ_1/ (Schweitzer et al., 2014). Then, sequenced on a NextSeq500 mid lane output PE 150bp. A custom sequencing recipe was run; read 1 [36 imaged cycles], index 1 [8 imaged cycles, 27 dark cycles, 8 imaged cycles], index 2 [8 imaged cycles, 21 dark cycles, 8 imaged cycles], read 2 [36 imaged cycles].

\section{Data pre-processing}

Libraries were preprocessed and aligned as in Preissl et al. Reads were aligned to the $\mathrm{mm} 10$ genome using Bowtie2 in paired-end mode (-p 5 -t -X2000 --no-mixed --no-discordant -mm) then duplicated reads and reads with $M A P Q<30$ were removed. The doublet score and TSS enrichment score were then calculated using ArchR (Granja et al., 2021). After removing doublets, barcodes with fewer than 1000 fragments and a TSS score of less than 4 were removed.

\section{Computational analysis of snATAC-seq data}

The ArchR analysis pipeline was used for most of the data analysis. After doublet removal and quality control cutoffs, dimensionality reduction was performed using ArchR's LSI-SVD method. A 10kb tile matrix was used for clustering and UMAP visualization. For cell type identification, ArchR's getMarkerFeatures() was used to find differentially accessible genes for each cluster. The gene score count matrix was used for all gene accessibility analyses. GO enrichment was performed by ClusterProfiler's compareCluster with a p-value cutoff of 0.05 . MAGIC was used for gene accessibility imputation for gene score visualization of specific marker genes. Peaks were identified using default parameters in ArchR, which call peaks using MACS2 on each cluster, then all peaks were merged using ArchR's iterative merging approach. For motif enrichment, ArchR's hypergeometric enrichment test peakAnnoEnrichment() was used with motif annotations from the CIS-BP motif database.

\section{GR motifs and GR ChIP-seq comparison}

Data from Yu et al. 2010 was downloaded from GEO (GSE24105). The ChIP-seq peaks were then lifted over from mm9 to mm10 annotation. Any overlap with the top 3,000 peaks from INGVSG and ING-SHAM were counted.

\section{Fatty acid methyl ester identification and quantitative profile}

Fatty acid methyl esters (FAME) were prepared using a modified one-step method of Garces and Mancha (Garcés \& Mancha, 1993). FAME were identified and quantified by Shimadzu 
GCMS-TQ8050 triple quadrupole mass spectrometer with a CI-MS (Shimadzu, Kyoto, Japan) with a BPX 70 column $(25 \mathrm{~m} \times 0.22 \mathrm{~mm} \times 0.25 \mu \mathrm{m}$; SG

E Inc., Austin, TX). An equal weight FAME mixture (462A; Nu-Chek Prep, Inc.) was used to calculate response factors daily. GC conditions: The program was $30 \mathrm{~min}$ in total, the column oven temperature was initially held at $80^{\circ} \mathrm{C}$ and then ramped at the rate of $15^{\circ} \mathrm{C} / \mathrm{min}$ to $170^{\circ} \mathrm{C}$ and held for $4 \mathrm{~min}$, then ramped to $240^{\circ} \mathrm{C}$ at the rate of $7^{\circ} \mathrm{C} / \mathrm{min}$ and held for $10 \mathrm{~min}$. The injection temperature was at $250^{\circ} \mathrm{C}$ on a splitless injection mode with a 1.5 min solvent cut time. Helium was used as carrier gas in a linear velocity flow control mode, with pressure at $123.5 \mathrm{kPa}$, total flow at $30.0 \mathrm{~mL} / \mathrm{min}$, column flow at $1.31 \mathrm{~mL} / \mathrm{min}$, linear velocity at $46.2 \mathrm{~cm} / \mathrm{s}$, and purge flow at $5.0 \mathrm{~mL} / \mathrm{min}$. Argon was the collision gas and also used to pressurize the solvent-mediated chemical ionization source containing acetonitrile source maintained at 20 $\mathrm{kPa}$. Ion source and interface temperature were set at $240{ }^{\circ} \mathrm{C}$. 